\title{
Full-Wave Three-Dimensional Microwave Imaging With a Regularized Gauss-Newton Method- Theory and Experiment
}

\author{
Jürgen De Zaeytijd, Ann Franchois, Senior Member, IEEE, Christelle Eyraud, and Jean-Michel Geffrin
}

\begin{abstract}
A reconstruction algorithm is detailed for threedimensional full-vectorial microwave imaging based on Newtontype optimization. The goal is to reconstruct the three-dimensional complex permittivity of a scatterer in a homogeneous background from a number of time-harmonic scattered field measurements. The algorithm combines a modified Gauss-Newton optimization method with a computationally efficient forward solver, based on the fast Fourier transform method and the marching-on-in-source-position extrapolation procedure. A regularized cost function is proposed by applying a multiplicative-additive regularization to the least squares datafit. This approach mitigates the effect of measurement noise on the reconstruction and effectively deals with the non-linearity of the optimization problem. It is furthermore shown that the modified Gauss-Newton method converges much faster than the Broyden-Fletcher-Goldfarb-Shanno quasi-Newton method. Promising quantitative reconstructions from both simulated and experimental data are presented. The latter data are bi-static polarimetric free-space measurements provided by Institut Fresnel, Marseille, France.
\end{abstract}

Index Terms-Electromagnetic scattering, inverse problems, microwave imaging, optimization methods.

\section{INTRODUCTION}

$\mathbf{T}$ HE emerging techniques for full-wave three-dimensional (3-D) non-linear inversion of Maxwell's equations may contribute to major improvements in imaging performance from the microwave to the optical ranges and create new applications in fields as non-destructive testing, biomedical imaging and security. During the last two decades quantitative imaging techniques in the microwave region-providing reconstructions of electromagnetic material parameters as permittivity and conductivity - were mainly developed for two-dimensional (2-D), mostly transverse magnetic (TM)-polarized configurations [1]-[9]. In this 2-D scalar but non linearized-no Born approximation-framework various iterative reconstruction algorithms for different application-specific configurations [10],

Manuscript received December 22, 2006; revised June 13, 2007.

J. De Zaeytijd and A. Franchois are with the Department of Information Technology (INTEC-IMEC), Ghent University, B-9000 Gent Belgium (e-mail: jurgen.dezaeytijd@intec.ugent.be; ann.franchois@intec.ugent.be).

C. Eyraud was with the Institut Fresnel, UMR CNRSTIC 6133, Universite Paul Cézanne Aix-Marseille, III, Ecole Généraliste d'Ingénieurs de Marseille, Université de Provence Aix-Marseille I, 13392 Marseille Cedex, France. She is now with the Laboratoire de Planétologie, CNRS, Université Joseph Fourier, 38041 Grenoble Cedex, France.

J.-M. Geffrin is with the Institut Fresnel, UMR CNRSTIC 6133, Université Paul Cézanne Aix-Marseille, III, Ecole Généraliste d'Ingénieurs de Marseille, Université de Provence Aix-Marseille I, 13392 Marseille Cedex, France (e-mail: Jean-Michel.Geffrin@fresnel.fr).

Digital Object Identifier 10.1109/TAP.2007.908824
[11] as well as some experimental issues have been investigated [12]-[14]. Together with the advent of efficient numerical approaches and more powerful PCs, this is facilitating the full-wave 3-D quantitative inversion of strongly contrasted and/or inhomogeneous objects. A computationally demanding implementation of the Newton-Kantorovich algorithm was applied to a very simple 3-D configuration by Joachimowicz et al. [2] in 1991. More recently Bulyshev et al. [15] developed a 3-D microwave tomography approach in a scalar approximation and Abubakar et al. [16] presented full-vectorial 3-D inversion results using a multiplicative regularized contrast source inversion method, in both cases for biomedical applications. Other recent work regarding 3-D inversion is reported in [17], where full 3-D inversion is investigated on breast tissue, in [18], where the authors extend their recently developed 2-D multiscaling strategy to the 3-D case, in [19], where quantitative imaging is used for optical diffraction tomography and in [20], where 3-D quantitative inversion is performed in the time domain. In this paper we present a full-wave 3-D complex permittivity reconstruction algorithm for inhomogeneous lossy dielectric objects in a homogeneous background. Reconstructions are discussed for some simple 3-D homogeneous and inhomogeneous lossy dielectric objects with simulated data and for a dielectric cube with experimental data, collected from the bi-static polarimetric free-space measurement setup of Institut Fresnel, Marseille, France [21]. The data are different components of the scattered field vector in points surrounding the object, resulting from successive dipole illuminations with different polarizations and different source positions at a fixed frequency.

Since quantitative imaging involves the numerical solution of a non-linear inverse scattering problem, an iterative reconstruction algorithm is mandatory. The performance strongly depends on proper choices for a cost-function, a regularization strategy and an optimization scheme. In the 2-D case most authors employ a least squares error cost-function, which measures the distance between the measured field data and the scattered field computed via a numerical model, possibly augmented with a regularization term that accounts for a-priori information on the object. Since the scattered field depends on two types of unknowns, the complex permittivity and the total field inside the object, which are related by a domain integral equation constraint, two approaches for defining the cost-function have been reported. In the first, "conventional," approach, the total field unknown is eliminated by substitution, such that the cost-function only depends on the complex permittivity. This approach involves the solution of a full forward problem in each itera- 


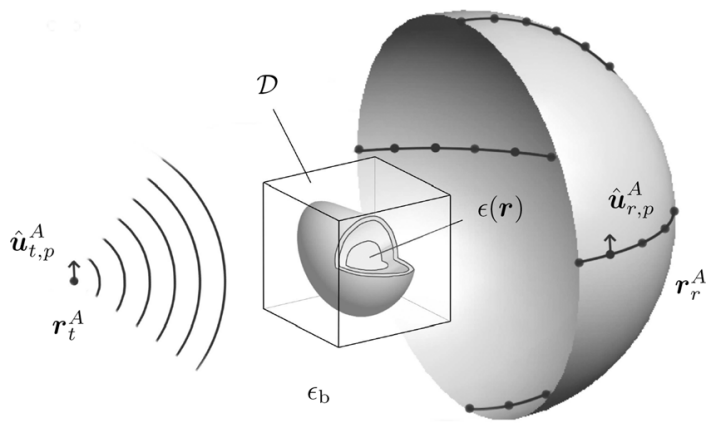

Fig. 1. The 3-D measurement configuration.

tion of the optimization. Various Newton-type schemes [1], [2], [5], [8], [11] and a few global optimization techniques [3], [7] have been applied here. In the second, "modified gradient," approach [4], [6], [9], the integral equation constraint is added as a second term to the cost-function, which then is optimized for both types of unknowns independently, using a conjugate-gradient optimization scheme. The main advantage of this approach is that no forward problem solutions are needed, but the number of optimization variables and iterations usually is much larger than with the conventional approach.

In this paper, the "conventional" approach is adopted and implemented with a fast forward solver and an efficient optimization scheme. The forward solver uses the FFT-method [22] to speed up the iterative solution of the multiview scattering problems and a "marching-on-in-angle" method [8] for an optimal choice of the initial estimates. Together with the fact that a high accuracy of the forward problem solution is not needed when the data are noisy, this approach may well be competitive in terms of computation time with the above mentioned "modified gradient" method, possibly depending on the transmitter/receiver configuration. As for the optimization, a cost-function with an additive-multiplicative regularization is proposed and minimized with a modified Gauss-Newton algorithm. This technique results in a rapid convergence and appears to outperform the Broyden-Fletcher-Goldfarb-Shanno (BFGS) quasi-Newton method [23]. The reconstruction results on both simulated and measured data are very promising.

In Section II, the 3-D inverse problem is formulated and the measurement configuration is defined. An efficient full-vectorial 3-D forward solution is described in Section III. In Section IV, a cost-function with additive-multiplicative regularization is proposed and a modified Gauss-Newton algorithm is presented. Some remarks on convergence and regularizing properties are made, without pursuing mathematical rigor. Finally, reconstructions from simulated and experimental data are presented in Section V.

\section{PROBLem Formulation}

A 3-D inhomogeneous, possibly lossy, dielectric object with arbitrary shape and complex permittivity $\epsilon(\boldsymbol{r})=\epsilon^{\prime}(\boldsymbol{r})-j \epsilon^{\prime \prime}(\boldsymbol{r})$, where $\boldsymbol{r}=x \hat{\boldsymbol{x}}+y \hat{\boldsymbol{y}}+z \hat{\boldsymbol{z}}$ denotes the position and $\hat{\boldsymbol{u}}$ is a unit vector in the $u$-direction, is sitting in a homogeneous medium with given complex permittivity $\epsilon_{\mathrm{b}}$, see Fig. 1. All materials are non-magnetic and have the permeability of free space $\mu_{0}$. We define a bounded domain $\mathcal{D}$ that completely includes the object and we propose to reconstruct $\epsilon(\boldsymbol{r})$ or, equivalently, the complex permittivity contrast

$$
\chi(\boldsymbol{r})=\epsilon(\boldsymbol{r})-\epsilon_{\mathrm{b}}
$$

everywhere within $\mathcal{D}$. Therefore, successive multiview illuminations with known time-harmonic incident fields $\boldsymbol{E}^{\text {inc }}$ are applied and the corresponding scattered fields $\boldsymbol{E}^{\text {scat }}$ are measured in points $\boldsymbol{r}^{\mathrm{A}}$ that belong to an observation domain outside $\mathcal{D}$. The time dependence $e^{j \omega t}$, with $\omega$ the angular frequency, is implicitly assumed.

We employ a contrast-source integral equation formulation, in which the scattered field $\boldsymbol{E}^{\text {scat }}$ is radiated by a contrast current density $\boldsymbol{J}^{\text {scat }}$ in the medium $\epsilon_{\mathrm{b}}$

$$
\boldsymbol{J}^{\text {scat }}(\boldsymbol{r})=j \omega \chi(\boldsymbol{r}) \boldsymbol{E}(\boldsymbol{r})
$$

which is zero everywhere outside the source volume $\mathcal{D}$ and where $\boldsymbol{E}=\boldsymbol{E}^{\text {inc }}+\boldsymbol{E}^{\text {scat }}$ is the total field. The vector contrast-source integral equation for the total field in an arbitrary point $\boldsymbol{r}$ can be written as [24], [22]

$$
\begin{aligned}
& \boldsymbol{E}(\boldsymbol{r})=-j \omega \mu_{0} {\left[\boldsymbol{I}+\frac{1}{k_{\mathrm{b}}^{2}} \nabla \nabla\right] } \\
& \cdot \int_{\mathbb{R}^{3}} G_{\mathrm{b}}\left(\boldsymbol{r}-\boldsymbol{r}^{\prime}\right)\left[\boldsymbol{J}^{\text {inc }}\left(\boldsymbol{r}^{\prime}\right)+\boldsymbol{J}^{\mathrm{scat}}\left(\boldsymbol{r}^{\prime}\right)\right] \mathrm{d} \boldsymbol{r}^{\prime},
\end{aligned}
$$

where $\boldsymbol{I}$ is the $3 \times 3$ identity matrix and $k_{\mathrm{b}}=\omega \sqrt{\epsilon_{\mathrm{b}} \mu_{0}}$ is the propagation constant of the background medium. $\boldsymbol{J}^{\text {inc }}$ with support $\mathcal{S} \not \subset D$ is the source current density for $\boldsymbol{E}^{\text {inc }}$ and

$$
G_{\mathrm{b}}\left(\boldsymbol{r}-\boldsymbol{r}^{\prime}\right)=\frac{e^{-j k_{\mathrm{b}}\left\|\boldsymbol{r}-\boldsymbol{r}^{\prime}\right\|}}{4 \pi\left\|\boldsymbol{r}-\boldsymbol{r}^{\prime}\right\|}
$$

is the scalar Green's function of homogeneous space, which satisfies

$$
\nabla^{2} G_{\mathrm{b}}\left(\boldsymbol{r}-\boldsymbol{r}^{\prime}\right)+k_{\mathrm{b}}^{2} G_{\mathrm{b}}\left(\boldsymbol{r}-\boldsymbol{r}^{\prime}\right)=-\delta\left(\boldsymbol{r}-\boldsymbol{r}^{\prime}\right) .
$$

As usual, the non-linear inverse scattering problem is described by two equations: a domain integral equation

$$
\boldsymbol{E}^{\mathrm{inc}}(\boldsymbol{r})=\boldsymbol{E}(\boldsymbol{r})+j \omega \boldsymbol{A}^{\mathrm{scat}}(\boldsymbol{r})+\nabla \varphi^{\mathrm{scat}}(\boldsymbol{r})
$$

for $\boldsymbol{r}$ belonging to $\mathcal{D}$, and an observation equation

$$
\boldsymbol{E}^{\text {scat }}\left(\boldsymbol{r}^{\mathrm{A}}\right)=\frac{k_{\mathrm{b}}^{2}}{\epsilon_{\mathrm{b}}} \int_{\mathcal{D}} \boldsymbol{G}_{\mathrm{b}}\left(\boldsymbol{r}^{\mathrm{A}}-\boldsymbol{r}^{\prime}\right) \cdot \chi\left(\boldsymbol{r}^{\prime}\right) \boldsymbol{E}\left(\boldsymbol{r}^{\prime}\right) d \boldsymbol{r}^{\prime}
$$

which relates the scattered field in the observation points to the unknown contrast sources. Note that (7) is non-linear in $\chi$ since $\boldsymbol{E}$ depends on $\chi$ by (6). In (6), the expression of the scattered field in terms of vector and scalar potentials $\boldsymbol{A}^{\text {scat }}$ and $\varphi^{\text {scat }}$ is employed to facilitate the numerical solution of the 3 -D vectorial forward problem [25]. The tensor $\boldsymbol{G}_{\mathrm{b}}$ in (7) is the dyadic Green's function of homogeneous space, such that the field generated by an elementary dipole oriented along a unit vector $\hat{\boldsymbol{u}}$ with current density

$$
\boldsymbol{J}_{\delta}(\mathbf{r})=-\frac{1}{j \omega \mu_{0}} \delta\left(\boldsymbol{r}-\boldsymbol{r}^{\prime}\right) \hat{\boldsymbol{u}}
$$

in homogeneous space is given by $\boldsymbol{G}_{\mathrm{b}}\left(\boldsymbol{r}-\boldsymbol{r}^{\prime}\right) \cdot \hat{\boldsymbol{u}}$. 


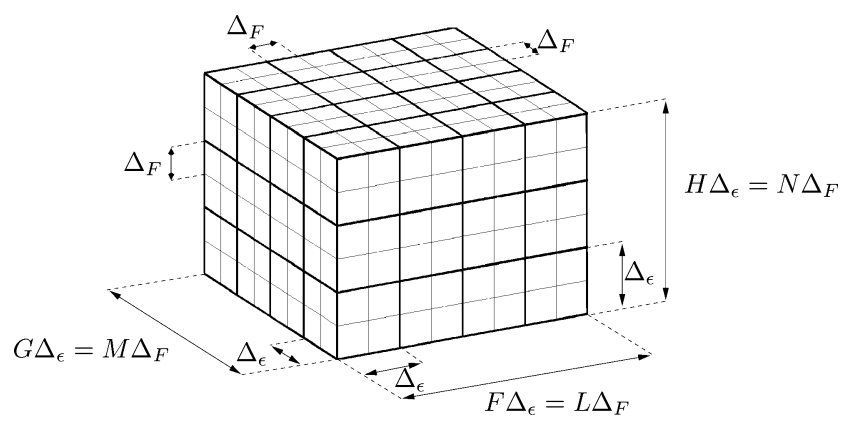

Fig. 2. Definitions for the grids of the inverse and the direct problem.

For the numerical reconstruction, it is necessary to parameterize the unknown permittivity $\epsilon(\boldsymbol{r})$ in $\mathcal{D}$. Therefore the domain $\mathcal{D}$ is contained in a uniform grid $\mathcal{D}^{\epsilon}$ with $N^{\epsilon}$ cubic cells with side $\Delta_{\epsilon}$. The shape of this parameter grid $\mathcal{D}^{\epsilon}$ can be chosen arbitrarily provided $\mathcal{D} \subset \mathcal{D}^{\epsilon}$, but in this paper it is a cuboid with $N^{\epsilon}=F \times G \times H$ cells in the $x^{-}, y^{-}$, and $z$-directions, respectively (Fig. 2). The gridpoints then are denoted by $\boldsymbol{r}_{f, g, h}=$ $f \Delta_{\epsilon} \hat{\boldsymbol{x}}+g \Delta_{\epsilon} \hat{\boldsymbol{y}}+h \Delta_{\epsilon} \hat{\boldsymbol{z}}$. The permittivity is approximated with a piecewise constant function that has one value in each cell

$$
\epsilon(\boldsymbol{r}) \approx \sum_{f=0}^{F-1} \sum_{g=0}^{G-1} \sum_{h=0}^{H-1} \epsilon_{f, g, h} \epsilon_{\mathrm{b}} \Phi_{f, g, h}(\boldsymbol{r})
$$

where $\Phi_{f, g, h}$ is a 3-D pulse function that assumes the value 1 inside cell $(f, g, h)$ and is zero elsewhere. The unknown coefficients $\epsilon_{f, g, h}$ in (9) represent relative complex permittivity values. They are collected in a $N^{\epsilon}$-dimensional complex permittivity vector

$$
\varepsilon=\left[\epsilon_{0,0,0} \ldots \epsilon_{F-1, G-1, H-1}\right]^{T}=\left[\epsilon_{\nu}\right]^{T}
$$

where $(\cdot)^{T}$ stands for transpose. The triplet $(f, g, h)$ is replaced with a single index $\nu$, whenever this is more convenient.

Let us denote the measured scattered field by $\boldsymbol{E}^{\text {meas }}$. In this paper the non-linear data (7) is solved for the complex permittivity vector $\varepsilon$ subject to the domain equation (6), in an iterative manner using Newton-type optimization methods. This is done by minimizing a cost-function, which includes a measure for the distance between the data $\boldsymbol{E}^{\text {meas }}$ and the model $\boldsymbol{E}^{\text {scat }}(\varepsilon)$ and which we discuss in more detail in Section IV. A full forward problem (6) and (7) is solved for each iterate $\varepsilon_{k}$ and update directions are computed from the derivatives of the scattered field. The derivation of a closed-form expression for the derivative of the scattered field is straightforward. To simplify the notations we introduce an operator $\mathcal{G}_{\mathrm{b}}^{\mathcal{V}}$ that acts on a vector function $\boldsymbol{p}$ with support $\mathcal{V}$

$$
\begin{aligned}
& {\left[\mathcal{G}_{\mathrm{b}}^{\mathcal{V}}(\boldsymbol{p})\right](\boldsymbol{r})=-j \omega \mu_{0}\left[\boldsymbol{I}+\frac{1}{k_{\mathrm{b}}^{2}} \nabla \nabla\right] } \\
& \cdot \int_{\mathcal{V}} G_{\mathrm{b}}\left(\boldsymbol{r}-\boldsymbol{r}^{\prime}\right) \boldsymbol{p}\left(\boldsymbol{r}^{\prime}\right) \mathrm{d} \boldsymbol{r}^{\prime} .
\end{aligned}
$$

Using this operator notation, we write the total field (3) in an arbitrary point $r$ as

$$
\boldsymbol{E}(\boldsymbol{r})=\left[\mathcal{G}_{\mathrm{b}}^{\mathcal{S}}\left(\boldsymbol{J}^{\mathrm{inc}}\right)\right](\boldsymbol{r})+\left[\mathcal{G}_{\mathrm{b}}^{\mathcal{D}}(j \omega \chi \boldsymbol{E})\right](\boldsymbol{r}) .
$$

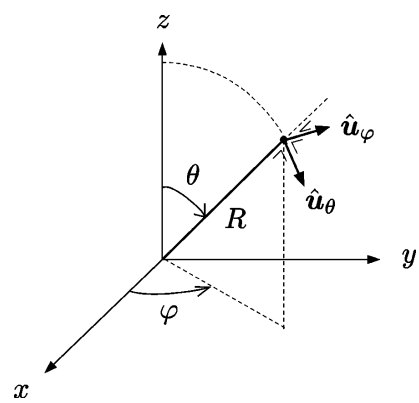

Fig. 3. Notations concerning spherical coordinates.

We also define a dyadic Green's function of the inhomogeneous medium $G_{\text {inh }}$, such that $G_{\text {inh }}\left(r, r^{\prime}\right) \cdot \hat{\boldsymbol{u}}$ corresponds to the total field in $\boldsymbol{r}$ due to an elementary dipole (8) in $\boldsymbol{r}^{\prime}$ in presence of the scattering object. The total field (12) then also is given by

$$
\boldsymbol{E}(\boldsymbol{r})=-j \omega \mu_{0} \int_{\mathcal{S}} \boldsymbol{G}_{\mathrm{inh}}\left(\boldsymbol{r}, \boldsymbol{r}^{\prime}\right) \cdot \boldsymbol{J}^{\mathrm{inc}}\left(\boldsymbol{r}^{\prime}\right) \mathrm{d} \boldsymbol{r}^{\prime} .
$$

Taking the derivative of (7) —or equivalently of (12), since the incident field $\boldsymbol{E}^{\text {inc }}$ does not depend on the permittivity-with respect to the permittivity parameter $\epsilon_{\nu}$ yields

$$
\begin{aligned}
\frac{\partial \boldsymbol{E}^{\mathrm{scat}}(\boldsymbol{r})}{\partial \epsilon_{\nu}}=\left[\mathcal{G}_{\mathrm{b}}^{\mathcal{D}}\left(j \omega \epsilon_{b} \Phi_{\nu} \boldsymbol{E}\right)\right](\boldsymbol{r}) & \\
+ & {\left[\mathcal{G}_{\mathrm{b}}^{\mathcal{D}}\left(j \omega \chi \frac{\partial \boldsymbol{E}^{\mathrm{scat}}}{\partial \epsilon_{\nu}}\right)\right](\boldsymbol{r}) }
\end{aligned}
$$

where we have used (1) and (9). It follows that $\partial \boldsymbol{E}^{\text {scat }} / \partial \epsilon_{\nu}$ satisfies an equation as (12) with $\boldsymbol{J}^{\text {inc }}$ replaced with a current density $j \omega \epsilon_{b} \Phi_{\nu} \boldsymbol{E}$ in cell $\nu$. Following (13), the derivative of the scattered field thus can be written as

$$
\frac{\partial \boldsymbol{E}^{\mathrm{scat}}}{\partial \epsilon_{\nu}}(\boldsymbol{r})=k_{b}^{2} \int_{\mathcal{D}} \Phi_{\nu}\left(\boldsymbol{r}^{\prime}\right) \boldsymbol{G}_{\mathrm{inh}}\left(\boldsymbol{r}, \boldsymbol{r}^{\prime}\right) \cdot \boldsymbol{E}\left(\boldsymbol{r}^{\prime}\right) \mathrm{d} \boldsymbol{r}^{\prime} .
$$

For the scalar 3-D inverse scattering problem it is shown in [26] that a unique solution exists when the scattered far field is known in all directions for plane wave illuminations from all possible directions. In a real experiment the number of illuminations and measurements is finite and uniqueness cannot easily be established, but we assume that the problem of non-uniqueness can be alleviated by employing sufficient data. We consider a total number of $N^{\mathrm{A}}$ antenna positions, denoted with $\boldsymbol{r}_{a}^{\mathrm{A}}, a=1, \ldots, N^{\mathrm{A}}$, for the transmitting and receiving antennas. We choose a number of $N^{\mathrm{P}} \leq 3$ different polarizations and define for each antenna position $\boldsymbol{r}_{a}^{\mathrm{A}}$ the polarization directions $\hat{\boldsymbol{u}}_{a, p}^{\mathrm{A}}, p=1, \ldots, N^{\mathrm{P}}$. In a spherical measurement geometry, for example, such as the polarimetric bistatic facility at Institut Fresnel [21], the antenna positions typically lie on circles on a spherical surface and the polarization directions coincide with $\hat{\boldsymbol{u}}_{\theta}, \hat{\boldsymbol{u}}_{\phi}$ (Fig. 3). For the transmitter we choose an elementary dipole (8) in $\boldsymbol{r}_{t}^{\mathrm{A}}$ oriented along $\hat{\boldsymbol{u}}_{t, p}^{\mathrm{A}}$, where $\{t\}$ is a subset of $\{a\}$. The total number of illuminations thus is given by the number of transmitter positions $N^{\mathrm{T}}$ times the number of polarizations $N^{\mathrm{P}}$. The field is measured in $N_{t}^{\mathrm{R}}$ receiver positions $\boldsymbol{r}_{r}^{\mathrm{A}}$ along the polarization directions $\hat{\boldsymbol{u}}_{r, p}^{\mathrm{A}}$, where the subset $\{r\}$ - and possibly $N_{t}^{\mathrm{R}}$ - usually is different for each illumination $t$. We collect the 
total amount of measured scattered field data in a vector $\boldsymbol{e}^{\text {meas }}$ with dimension $N^{\mathrm{D}}=\left(N^{\mathrm{P}}\right)^{2} \sum_{t} N_{t}^{\mathrm{R}} \leq\left(N^{\mathrm{P}}\right)^{2}\left(N^{\mathrm{A}}\right)^{2}$. This vector contains the scattered field components $\boldsymbol{E}_{t, p}^{\text {meas }}\left(\boldsymbol{r}_{r}^{\mathrm{A}}\right) \cdot \hat{\boldsymbol{u}}_{r, p^{\prime}}^{\mathrm{A}}$ in points $\boldsymbol{r}_{r}^{\mathrm{A}}$ and polarized along $p^{\prime}$ resulting from $p$-polarized dipole excitations in $\boldsymbol{r}_{t}^{\mathrm{A}}$. Similarly, the scattered field components $\boldsymbol{E}_{t, p}^{\mathrm{scat}}\left(\boldsymbol{r}_{r}^{\mathrm{A}}\right) \cdot \hat{\boldsymbol{u}}_{r, p^{\prime}}^{\mathrm{A}}$, which are computed for a given permittivity vector $\varepsilon$, are collected in a $N^{\mathrm{D}}$-dimensional vector $\boldsymbol{e}^{\text {scat }}$. The derivatives of these components with respect to $\epsilon_{\nu}$ are obtained from (15)

$$
\begin{aligned}
\frac{\partial \boldsymbol{E}_{t, p}^{\mathrm{scat}}}{\partial \epsilon_{\nu}}\left(\boldsymbol{r}_{r}^{\mathrm{A}}\right) \cdot \hat{\boldsymbol{u}}_{r, p^{\prime}}^{\mathrm{A}}=k_{b}^{2} \int_{\mathcal{D}} \Phi_{\nu}\left(\boldsymbol{r}^{\prime}\right) \hat{\boldsymbol{u}}_{r, p^{\prime}}^{\mathrm{A}} & \cdot \boldsymbol{G}_{\mathrm{inh}}\left(\boldsymbol{r}_{r}^{\mathrm{A}}, \boldsymbol{r}^{\prime}\right) \cdot \boldsymbol{E}_{t, p}\left(\boldsymbol{r}^{\prime}\right) \mathrm{d} \boldsymbol{r}^{\prime}
\end{aligned}
$$

and since by reciprocity $G_{\mathrm{inh}}\left(\boldsymbol{r}_{r}^{\mathrm{A}}, \boldsymbol{r}^{\prime}\right)=G_{\text {inh }}^{T}\left(\boldsymbol{r}^{\prime}, \boldsymbol{r}_{r}^{\mathrm{A}}\right)$ and since

$$
\boldsymbol{E}_{r, p^{\prime}}\left(\boldsymbol{r}^{\prime}\right)=G_{\text {inh }}\left(\boldsymbol{r}^{\prime}, \boldsymbol{r}_{r}^{\mathrm{A}}\right) \cdot \hat{\boldsymbol{u}}_{r, p^{\prime}}^{\mathrm{A}}
$$

it follows that

$$
\frac{\partial \boldsymbol{E}_{t, p}^{\mathrm{scat}}}{\partial \epsilon_{\nu}}\left(\boldsymbol{r}_{r}^{\mathrm{A}}\right) \cdot \hat{\boldsymbol{u}}_{r, p^{\prime}}^{\mathrm{A}}=k_{b}^{2} \int_{\mathcal{D}} \Phi_{\nu}\left(\boldsymbol{r}^{\prime}\right) \boldsymbol{E}_{t, p}\left(\boldsymbol{r}^{\prime}\right) \cdot \boldsymbol{E}_{r, p^{\prime}}\left(\boldsymbol{r}^{\prime}\right) \mathrm{d} \boldsymbol{r}^{\prime}
$$

Consequently, the computation of the scattered field derivatives requires the solution of a forward problem (6) for excitations along all polarizations $\hat{\boldsymbol{u}}_{a, p}^{\mathrm{A}}$ in all antenna (transmitting and receiving) positions $\boldsymbol{r}_{a}^{\mathrm{A}}$, whereas for the computation of the cost function only the transmitting positions $\boldsymbol{r}_{t}^{\mathrm{A}}$ are involved. This amounts to $N^{\mathrm{A}} N^{\mathrm{P}}$ forward problems (6) for a given complex permittivity vector $\varepsilon$. The total number of forward problems thus easily grows large with a 3-D full-vectorial iterative imaging technique. However, it also follows that for a measurement configuration in which transmitters are actually used in all antenna positions - this is often a preferred configuration- $\boldsymbol{E}_{t, p}\left(\boldsymbol{r}^{\prime}\right)$ and $\boldsymbol{E}_{r, p^{\prime}}\left(\boldsymbol{r}^{\prime}\right)$ in (18) are already computed to evaluate the cost function, hence practically no extra effort is needed for the computation of the derivatives. This may be an advantage over the modified gradient approach, where increasing the number of transmitters heavily increases the workload [16].

The dimension of a single forward problem often amounts to over tens of thousands of unknowns. Therefore, efficients algorithms are needed for economic use of memory and computation time.

\section{THE FORWARD PROBLEM}

For the numerical solution of the forward problem (6)-(7) a Galerkin method of moments is applied. We summarize here the main ideas and refer to [25] for a detailed description of the implementation. As in [22] $N^{\mathrm{B}}$ vectorial rooftop functions are used to test the domain equation (6) and to expand the electric flux density $\boldsymbol{D}=\epsilon \boldsymbol{E}$ in this equation. These functions are defined on a uniform cuboidal grid $\mathcal{D}^{\mathrm{F}}$ with $N^{\mathrm{F}}=L \times$ $M \times N$ cubic cells with side $\Delta_{\mathrm{F}}$, which is an integer subdivision of the cell side $\Delta_{\epsilon}$ of the parameter grid $\mathcal{D}^{\epsilon}$ (Fig. 2). The resulting $N^{\mathrm{B}}$-dimensional linear system is solved iteratively with the stabilized bi-conjugate gradient (BICGSTAB) method [27]. Since the uniform discretization preserves the convolutional property of the integral equation, the matrix-vector multiplications needed by the iterative solver can be accelarated with the use of fast Fourier transforms [22], [29], [28]. The resulting BICGSTAB-FFT method has a computational complexity of $\mathcal{O}\left(P N^{\mathrm{B}} \log N^{\mathrm{B}}\right)$, with $\mathrm{P}$ the number of iterations needed to reach the desired accuracy, and the memory complexity is $\mathcal{O}\left(N^{\mathrm{B}}\right)$.

In our inversion scheme the forward problem has to be solved several times: for varying contrast parameter configurations and with each of those for varying source positions and polarizations. An adequate choice of the initial guess can accelerate the iterative solution of the linear systems. Since in a practical measurement setup the transmitter is usually moved to successive positions $\boldsymbol{r}_{t}^{\mathrm{A}}$ in small steps, it is expected that the field varies accordingly in a smooth way. Let us formally write the discretized forward problem for a transmitter in $\boldsymbol{r}_{t}^{\mathrm{A}}$ as

$$
e_{t}^{\text {inc }}=Z d_{t}
$$

where the $N^{\mathrm{B}}$-dimensional vector $\boldsymbol{d}_{t}$ contains the coefficients of the discretized electric flux density and where $\boldsymbol{e}_{t}^{\text {inc }}$ contains the weighted incident field. With the marching-on-in-source-position technique [30], [8], the initial guess $\boldsymbol{d}_{t}^{0}$ is an extrapolation of solutions $\boldsymbol{d}_{t-1}, \boldsymbol{d}_{t-2}, \ldots$ obtained for $I$-typically 3 - previous transmitter positions

$$
\boldsymbol{d}_{t}^{0}=\sum_{i=1}^{I} a_{t, i} \boldsymbol{d}_{t-i}
$$

The coefficients $a_{t, i}$ are chosen such that the error $\left\|Z \boldsymbol{d}_{t}^{0}-e_{t}^{\mathrm{inc}}\right\|^{2}$ is minimized, hence they are a solution of

$$
\sum_{i=1}^{I}\left[e_{t-j}^{\mathrm{inc}}\right]^{H} e_{t-i}^{\mathrm{inc}} a_{t, i}=\left[\boldsymbol{e}_{t-j}^{\mathrm{inc}}\right]^{H} \boldsymbol{e}_{t}^{\mathrm{inc}} j=1, \ldots, I .
$$

\section{THE INVERSE PROBLEM}

\section{A. The Regularized Cost-Function}

We propose the following cost-function with an additive-multiplicative regularization

$$
\left.\mathcal{F}(\varepsilon)=\mathcal{F}^{\mathcal{L}}{ }^{(\varepsilon)\left[1+\alpha \mathcal{F}^{\mathcal{R}}\right.}(\varepsilon)\right]
$$

where $\mathcal{F}^{\mathcal{L}}$ is the least-squares data error, $\mathcal{F}^{\mathcal{R}}$ is a regularizing function, $\alpha$ is a regularization parameter and $\varepsilon$ is the complex permittivity vector (10). The least-squares data error is defined as

$$
\mathcal{F}^{\mathcal{L S}}(\varepsilon)=\frac{1}{\mathcal{N} \mathcal{L S}}\left\|\boldsymbol{e}^{\mathrm{scat}}(\varepsilon)-\boldsymbol{e}^{\text {meas }}\right\|^{2}
$$

with $\mathcal{N}^{\mathcal{L S}}=\left\|e^{\text {meas }}\right\|^{2}$ a normalization constant and $\|\cdot\|$ is the $L^{2}$ norm. The least-squares data error (23) has been used as a cost-function by many authors, but its minimum generally is not well-defined due to the ill-posedness of the inverse problem. Consequently, a sufficiently coarse grid $\mathcal{D}^{\epsilon}$ for the parameterization (9) of $\epsilon(\boldsymbol{r})$ is often chosen to reduce unwanted fluctuations in the reconstruction when the data are noisy. In this paper 
we apply a regularization by adding to (23) a multiplicative regularization term $\alpha \mathcal{F}^{\mathcal{L}} \mathcal{F}^{\mathcal{R}}$, where $\alpha$ is a small positive number and $\mathcal{F}^{\mathcal{R}}$ is the positive function

$$
\begin{aligned}
\mathcal{F}^{\mathcal{R}}(\varepsilon)= & \frac{1}{\mathcal{N}^{\mathcal{R}}}\left[\sum_{f=0}^{F} \sum_{g=0}^{G-1} \sum_{h=0}^{H-1}\left|\epsilon_{f, g, h}-\epsilon_{f-1, g, h}\right|^{2}\right. \\
& +\sum_{f=0}^{F-1} \sum_{g=0}^{G} \sum_{h=0}^{H-1}\left|\epsilon_{f, g, h}-\epsilon_{f, g-1, h}\right|^{2} \\
& \left.+\sum_{f=0}^{F-1} \sum_{g=0}^{G-1} \sum_{h=0}^{H}\left|\epsilon_{f, g, h}-\epsilon_{f, g, h-1}\right|^{2}\right]
\end{aligned}
$$

with $\mathcal{N}^{\mathcal{R}}$ a normalization constant, which accounts for the dimensions of the object and the discretization cell. The optimization in this paper will start from a constant relative permittivity $\epsilon^{\text {init }}$ for all cells of $\mathcal{D}^{\epsilon}$ and whenever the triplet $(f, g, h)$ in (24) indicates a cell just outside $\mathcal{D}^{\epsilon}$, the corresponding value of $\epsilon_{f, g, h}$ is also taken equal to $\epsilon^{\text {init }}$. The function $\mathcal{F}^{\mathcal{R}}$ is a discrete version of $(1 / \mathcal{D}) \int_{\mathcal{D}}|\nabla \epsilon(\boldsymbol{r})|^{2} \mathrm{~d} \boldsymbol{r}$, hence it penalizes strong local fluctuations in the permittivity. Note that this regularization resembles the one used in [31], where the principle of total variation is incorporated as a multiplicative regularization to a modified gradient cost-function.

The cost-function $\mathcal{F}$ is always larger than the data error $\mathcal{F} \mathcal{L}$, except when $\mathcal{F}^{\mathcal{L}}=0$, i.e., at the solution in absence of measurement noise. For exact data the regularized solution thus automatically coincides with the exact solution. Although in this case regularization is not strictly necessary, the term $\alpha \mathcal{F}^{\mathcal{L}} \mathcal{F}^{\mathcal{R}}$ appears to have a positive effect on the convergence of the optimization algorithm, in terms of coping with the non-linearity. When the data are noisy, the minimization of $\mathcal{F}$ is stopped when the data error $\mathcal{F}^{\mathcal{L S}}$ reaches the noise level $h$, i.e., $\mathcal{F}^{\mathcal{L S}} \leq h$. The regularization then provides a smoothed solution, dependent on the value of $\alpha$. This value is a compromise between the desired amount of smoothing and the capability to reconstruct strongly inhomogeneous objects. As observed from numerical experiments with the multiplicative additive regularization, the reconstruction is not very sensitive to the specific choice of $\alpha$, as long as it allows the data error $\mathcal{F}^{\mathcal{L}}$ to reach the noise level. This condition is known as the discrepancy principle [32]. Usually we choose $\alpha \mathcal{F}^{\mathcal{R} 0} \approx 10^{-3}$ to $10^{-2}$, with $\mathcal{F}^{\mathcal{R} 0}$ the value of $\mathcal{F}^{\mathcal{R}}$ at the true solution. An order of magnitude of $\mathcal{F}^{\mathcal{R} 0}$ can be deduced from very general a priori information such as the maximum size of the object (as used to choose a domain $\mathcal{D}$ ) and an upper limit for the permittivity (as used to choose a cell size for the forward problem). A mathematical proof of the regularizing properties of the proposed method is not given, but some further insight is provided in Section IV-D.

\section{B. The Optimization: General}

Newton's method for local optimization approximates a nonlinear cost-function with a quadratic model based on the function's first and second order derivatives (the gradient and the Hessian matrix) at the current iterate and chooses the stationary point (minimum) of this model as the next iterate. It has the attractive fundamental property of super-linear convergence if the initial guess is close enough to the solution [23]. However, when starting further away from the solution, the Newton correction may lead to an increase in the cost function, if at its stationary point the model is no longer a good approximation to the function or if the Hessian matrix is not positive definite. Furthermore, for large optimization problems the computation of second order derivatives is usually very expensive. A variety of Newton-type methods that try to deal with these issues are described in the literature. In this paper we propose a modified Gauss-Newton method and compare it to a BFGS quasi-Newton method. Both methods are combined with a line search to enlarge the convergence domain.

Let us first give a few definitions. The cost-function $\mathcal{F}(\varepsilon)$ and the functions $\mathcal{F}^{\mathcal{L}}(\varepsilon)$ and $\mathcal{F}^{\mathcal{R}}(\varepsilon)$ are real functions of $N^{\epsilon}$ complex variables $\epsilon_{\nu}=\epsilon_{\nu}^{\prime}-j \epsilon_{\nu}^{\prime \prime}$. We define a gradient

$$
\boldsymbol{g}=\left[\begin{array}{l}
\frac{\partial \mathcal{F}}{\partial \epsilon_{\nu}} \\
\frac{\partial \mathcal{F}}{\partial \epsilon_{\nu}^{*}}
\end{array}\right]
$$

and a Hessian matrix

$$
\boldsymbol{H}=\left[\begin{array}{cc}
\frac{\partial^{2} \mathcal{F}}{\partial \epsilon_{\nu} \partial \epsilon_{v}} & \frac{\partial^{2} \mathcal{F}}{\partial \epsilon_{\nu} \partial \epsilon_{v}^{*}} \\
\frac{\partial^{2} \mathcal{F}}{\partial \epsilon_{\nu}^{*} \partial \epsilon_{v}} & \frac{\partial^{2} \mathcal{F}}{\partial \epsilon_{\nu}^{*} \partial \epsilon_{v}^{*}}
\end{array}\right]
$$

with derivatives with respect to $\epsilon_{\nu}$ and its complex conjugate $\epsilon_{\nu}^{*}$, which are considered as independent variables, and with $\nu, v=1 \ldots N^{\epsilon}$. These definitions are equivalent with the use of $\epsilon_{\nu}^{\prime}$ and $\epsilon_{\nu}^{\prime \prime}$ as independent variables [33], and lead to simplified notations in the following. It is known for sums of squares that the gradient and Hessian matrix take particular forms. For the least-squares data error $\mathcal{F}^{\mathcal{L}}$ the gradient is

$$
\boldsymbol{g}^{\mathcal{L S}}=\frac{1}{\mathcal{N} \mathcal{L S}}\left[\begin{array}{c}
\boldsymbol{J}^{T}\left[\boldsymbol{e}^{\text {scat }}-\boldsymbol{e}^{\text {meas }}\right]^{*} \\
\boldsymbol{J}^{H}\left[\boldsymbol{e}^{\text {scat }}-e^{\text {meas }}\right]
\end{array}\right]
$$

where $\boldsymbol{J}$ is the $N^{\mathrm{D}} \times N^{\epsilon}$ Jacobian matrix containing the first order derivatives of the scattered field components, $J_{d \nu}=\partial e_{d}^{\mathrm{scat}} / \partial \epsilon_{\nu}$, and the Hessian matrix is

$$
\boldsymbol{H}^{\mathcal{L S}}=\frac{1}{\mathcal{N} \mathcal{L S}}\left[\begin{array}{cc}
\boldsymbol{B} & \boldsymbol{J}^{T} \boldsymbol{J}^{*} \\
\boldsymbol{J}^{H} \boldsymbol{J} & \boldsymbol{B}^{*}
\end{array}\right]
$$

where $\boldsymbol{B}$ is a $N^{\epsilon} \times N^{\epsilon}$ matrix containing products of second order derivatives of the scattered field with the data residus, $B_{\nu v}=\left(\partial^{2} \boldsymbol{e}^{\mathrm{scat}} / \partial \epsilon_{\nu} \partial \epsilon_{v}\right)^{T}\left[\boldsymbol{e}^{\mathrm{scat}}-\boldsymbol{e}^{\text {meas }}\right]^{*}$. For the regularizing function $\mathcal{F}^{\mathcal{R}}$ we denote

$$
\boldsymbol{g}^{\mathcal{R}}=\left[\begin{array}{c}
\Omega \\
\Omega^{*}
\end{array}\right]
$$

with $\Omega_{\nu}=\partial \mathcal{F}^{\mathcal{R}} / \partial \epsilon_{\nu}$ and

$$
\boldsymbol{H}^{\mathcal{R}}=\left[\begin{array}{cc}
0 & \boldsymbol{\Sigma} \\
\boldsymbol{\Sigma} & 0
\end{array}\right]
$$

where $\boldsymbol{\Sigma}$ is a real and constant matrix with $\Sigma_{\nu v}=$ $\partial^{2} \mathcal{F}^{\mathcal{R}} / \partial \epsilon_{\nu} \partial \epsilon_{v}^{*}$. Consequently, the gradient and Hessian matrix of the regularized cost-function $\mathcal{F}$ can be written as

$$
\boldsymbol{g}=\boldsymbol{g}^{\mathcal{L S}}\left(1+\alpha \mathcal{F}^{\mathcal{R}}\right)+\mathcal{F}^{\mathcal{L S}} \alpha \boldsymbol{g}^{\mathcal{R}}
$$

and

$$
\boldsymbol{H}=\frac{1}{\mathcal{N}^{\mathcal{L S}}}\left[\begin{array}{cc}
\boldsymbol{B}^{\prime} & \boldsymbol{A}^{*} \\
\boldsymbol{A} & \boldsymbol{B}^{\prime *}
\end{array}\right]
$$


where $\boldsymbol{H}$ is a complex symmetric matrix with

$$
\begin{aligned}
\boldsymbol{B}^{\prime}= & \boldsymbol{B}\left(1+\alpha \mathcal{F}^{\mathcal{R}}\right)+\alpha \boldsymbol{J}^{T}\left[\boldsymbol{e}^{\text {scat }}-\boldsymbol{e}^{\text {meas }}\right]^{*} \boldsymbol{\Omega}^{T} \\
& +\alpha \boldsymbol{\Omega}\left[\boldsymbol{e}^{\text {scat }}-\boldsymbol{e}^{\text {meas }}\right]^{H} \boldsymbol{J}, \\
\boldsymbol{A}= & \boldsymbol{J}^{H} \boldsymbol{J}\left(1+\alpha \mathcal{F}^{\mathcal{R}}\right)+\alpha \boldsymbol{J}^{H}\left[\boldsymbol{e}^{\text {scat }}-\boldsymbol{e}^{\text {meas }}\right] \boldsymbol{\Omega}^{T} \\
& +\alpha \boldsymbol{\Omega}^{*}\left[\boldsymbol{e}^{\text {scat }}-\boldsymbol{e}^{\text {meas }}\right]^{H} \boldsymbol{J}+\alpha \mathcal{N}^{\mathcal{L S}} \mathcal{F}^{\mathcal{L S}} \boldsymbol{\Sigma} .
\end{aligned}
$$

In an optimization scheme the complex permittivity vector is iteratively updated as

$$
\varepsilon_{k+1}=\varepsilon_{k}+\Delta \varepsilon_{k}
$$

where $\Delta \varepsilon_{k}$ is the permittivity correction at iterate $k$. The Newton correction for $\mathcal{F}$ in complex notation then is given by

$$
\left[\begin{array}{l}
\Delta \varepsilon_{k} \\
\Delta \varepsilon_{k}^{*}
\end{array}\right]=-\boldsymbol{H}_{k}^{-1} \boldsymbol{g}_{k}
$$

This correction can then be used as a search direction along which an approximate line search is performed with an algorithm as described in [23]. In [23] it is also proven that an optimization method that searches successively along different descent paths using the aforementioned approximate line search converges to a (local) minimizer provided that the search directions are uniformly bounded away from orthogonality with the steepest descent direction. In this paper we always perform a line search after determining an update direction.

\section{The Gauss-Newton Method Applied to the Least Squares Data Error}

A widely used alternative for the Newton method in case of sums of squares of non-linear functions, such as the data error $\mathcal{F} \mathcal{L S}$, is the Gauss-Newton method. It consists of linearizing the function $\boldsymbol{e}^{\text {scat }}$ in (23) around the current iterate as

$$
\Delta e_{k}^{\text {scat }}=e^{\text {scat }}\left(\varepsilon_{k}+\Delta \varepsilon_{k}\right)-e_{k}^{\text {scat }}\left(\varepsilon_{k}\right) \approx J_{k} \Delta \varepsilon_{k} .
$$

From (37), by identifying $e^{\text {scat }}\left(\varepsilon_{k}+\Delta \varepsilon_{k}\right)=e^{\text {meas }}$ and by solving the resulting system in the least squares sense, the Gauss-Newton correction for $\mathcal{F}^{\mathcal{L} \mathcal{S}}$ is given by

$$
\Delta \varepsilon_{k}=-\left(\boldsymbol{J}_{k}^{H} \boldsymbol{J}_{k}\right)^{-1} \boldsymbol{J}_{k}^{H}\left[\boldsymbol{e}_{k}^{\text {scat }}-\boldsymbol{e}^{\text {meas }}\right] .
$$

Equation (38) is obtained as well from the Newton correction for $\mathcal{F L S}^{\mathcal{L}}$ by neglecting the matrix $\boldsymbol{B}$ in the Hessian matrix (28). No second order derivatives of the scattered fields thus are needed. Also, the hermitian matrix $\boldsymbol{J}^{H} \boldsymbol{J}$ is at least positive semi-definite, hence the update direction (38) is never uphill. However, the linearization (37) may be too bad an approximation far from the solution and the condition number of $\boldsymbol{J}^{H} \boldsymbol{J}$ typically is large due to the small singular values of $\boldsymbol{J}$. This numerical rank deficiency can prevent convergence, because update-directions can become almost orthogonal to the steepest descent vector. In [5] the convergence of the Gauss-Newton method was improved with a Levenberg-Marquardt trust-region approach, which ensures a positive definite and better conditioned approximate Hessian matrix.

\section{The Modified Gauss-Newton Method Applied to the Regularized Cost-Function}

In this paper we focus on the regularized cost-function $\mathcal{F}$. Applying the Newton formula (36) to this cost-function yields (the index $k$ is omitted in the following)

$$
\begin{aligned}
& \boldsymbol{A} \Delta \varepsilon+\boldsymbol{B}^{\prime *} \Delta \varepsilon^{*}=-\boldsymbol{J}^{H}\left[\boldsymbol{e}^{\text {scat }}-\boldsymbol{e}^{\text {meas }}\right]\left(1+\alpha \mathcal{F}^{\mathcal{R}}\right) \\
&+\alpha \mathcal{N}^{\mathcal{L}} \mathcal{F}^{\mathcal{L} \mathcal{S}} \boldsymbol{\Omega}^{*} .
\end{aligned}
$$

Using (33) and (34) and introducing the linearization (37), we obtain

$$
\begin{aligned}
\boldsymbol{A} \Delta \varepsilon & +\boldsymbol{B}^{*} \Delta \boldsymbol{\varepsilon}^{*} \\
= & \boldsymbol{J}^{H} \boldsymbol{J}\left(1+\alpha \mathcal{F}^{\mathcal{R}}\right) \Delta \varepsilon \\
& +\alpha \boldsymbol{J}^{H}\left[\boldsymbol{e}^{\text {scat }}-\boldsymbol{e}^{\text {meas }}\right]\left[\boldsymbol{\Omega}^{T} \Delta \varepsilon+\boldsymbol{\Omega}^{H} \Delta \varepsilon^{*}\right] \\
& +\alpha \boldsymbol{\Omega}^{*}\left[\left[\boldsymbol{e}^{\text {scat }}-\boldsymbol{e}^{\text {meas }}\right]^{H} \Delta \boldsymbol{e}^{\text {scat }}\right. \\
& \left.+\left[\boldsymbol{e}^{\text {scat }}-\boldsymbol{e}^{\text {meas }}\right]^{T} \Delta \boldsymbol{e}^{\text {scat* }}\right] \\
& +\alpha \mathcal{N}^{\mathcal{L} \mathcal{S}} \mathcal{F}^{\mathcal{L S}} \boldsymbol{\Sigma} \Delta \varepsilon .
\end{aligned}
$$

The optimization is started from a constant initial permittivity, i.e., $\epsilon_{f, g, h}=\epsilon^{\text {init }} \forall f, g, h$. This permittivity vector is the minimizer of $\mathcal{F}^{\mathcal{R}}$ and yields zero for $\Omega$. Consequently, the second and third terms in the right hand side of (40) are zero in the first iteration (and may remain small in a few subsequent iterations). The data residu $\Delta \boldsymbol{e}^{\text {meas }}=\left[\boldsymbol{e}^{\text {meas }}-\boldsymbol{e}^{\text {scat }}\right]$ eventually becomes small due to the minimization, such that all terms except the first one in the right hand side of (40) become negligible. Given this behavior at the beginning and end of the optimization, we choose to keep only the first and the last terms. This yields the modified Gauss-Newton correction

$$
\Delta \varepsilon=-\left(\boldsymbol{J}^{H} \boldsymbol{J}+\lambda^{2} \boldsymbol{\Sigma}\right)^{-1}\left(\boldsymbol{J}^{H}\left[\boldsymbol{e}^{\mathrm{scat}}-\boldsymbol{e}^{\mathrm{meas}}\right]+\lambda^{2} \boldsymbol{\Omega}^{*}\right)
$$

with $\lambda^{2}=\alpha \mathcal{N}^{\mathcal{L}} \mathcal{F}_{\mathcal{L}}^{\mathcal{L}} /\left(1+\alpha \mathcal{F}^{\mathcal{R}}\right)$.

The matrix $\boldsymbol{J}^{H} \boldsymbol{J}+\lambda^{2} \boldsymbol{\Sigma}$ in (41) is always positive definite for $\lambda^{2} \neq 0$, since $\boldsymbol{\Sigma}$ is positive definite. The presence of $\lambda^{2} \boldsymbol{\Sigma}$ thus ensures a descent direction. Since the optimization is stopped when $\mathcal{F}^{\mathcal{L}}$ reaches the noise level $h$ and with the reasonable assumption that $\mathcal{F}^{\mathcal{R}}$ is bounded by $\mathcal{F}_{\text {max }}^{\mathcal{R}}$ in the region of interest, a lower bound $\lambda_{\text {min }}^{2}=\alpha \mathcal{N}^{\mathcal{L} S} h /\left(1+\alpha \mathcal{F}_{\text {max }}^{\mathcal{R}}\right)$ exists for $\lambda^{2}$. Therefore, the update directions will be uniformly bounded away from orthogonality with the steepest descent vector along the optimization path and convergence to the stopping criterion or to a local minimum above this threshold can be established [23]. Furthermore, the more the data error is reduced, the more (41) approximates the Newton correction and super-linear convergence may be expected towards the end of the optimization.

As for the regularizing properties of this method, some remarks can be made by observing that the update (41) is the solution to the following regularized linear least squares problem:

$$
\min _{\Delta \varepsilon}\left\|\boldsymbol{J} \Delta \varepsilon-\Delta e^{\text {meas }}\right\|^{2}+\lambda^{2}\left\|L^{T} \Delta \varepsilon+L^{-1} \Omega^{*}\right\|^{2}
$$

where $\boldsymbol{L}$ is the Cholesky factor of $\boldsymbol{\Sigma}=\boldsymbol{L} \boldsymbol{L}^{T}$. The second term in (42) can be interpreted as a generalized Tikhonov regularization term [34]. It is minimized when $\boldsymbol{\Sigma} \Delta \varepsilon=-\boldsymbol{\Omega}^{*}(\varepsilon)$, hence also when $\Omega^{*}(\varepsilon+\Delta \varepsilon)=0$, since $\boldsymbol{\Sigma} \Delta \varepsilon=\Omega^{*}(\varepsilon+\Delta \varepsilon)-\Omega^{*}(\varepsilon)$. 
This term thus tries to reduce $\mathcal{F}^{\mathcal{R}}$, or fluctuations in the permittivity $\varepsilon$, which seems to be more appropriate than smoothing the correction $\Delta \varepsilon$, as was done by several authors with regular Tikhonov regularization, e.g., [1], [2], [5]. The regularization parameter $\lambda^{2}$ in (42) is proportional to the data error $\mathcal{F}^{\mathcal{L}}$ — see [2] for a similar strategy in case of regular Tikhonov regularization-hence the regularization term has a larger influence in the beginning of the minimization, which is beneficial for coping with the non-linearity.

\section{E. The BFGS Quasi-Newton Method Applied to the Regularized Cost-Function}

The quasi-Newton method consists in approximating the Hessian matrix in the Newton correction (36) with a matrix that does not involve the explicit computation of second order derivatives. In each iteration this approximated Hessian matrix is updated based on the change in the gradient with respect to the previous iteration. In this paper we apply the BFGS update formula for the inverse Hessian matrix $\boldsymbol{H}_{k}^{-1}$

$$
\begin{array}{r}
\tilde{\boldsymbol{H}}_{k+1}=\tilde{\boldsymbol{H}}_{k}+\left(1+\frac{\boldsymbol{\gamma}_{k}^{T} \tilde{\boldsymbol{H}}_{k} \boldsymbol{\gamma}_{k}}{\boldsymbol{\delta}_{k}^{T} \boldsymbol{\gamma}_{k}}\right) \frac{\boldsymbol{\delta}_{k} \boldsymbol{\delta}_{k}^{T}}{\boldsymbol{\delta}_{k}^{T} \boldsymbol{\gamma}_{k}} \\
-\left(\frac{\boldsymbol{\delta}_{k} \boldsymbol{\gamma}_{k}^{T} \tilde{\boldsymbol{H}}_{k}+\tilde{\boldsymbol{H}}_{k} \boldsymbol{\gamma}_{k} \boldsymbol{\delta}_{k}^{T}}{\boldsymbol{\delta}_{k}^{T} \boldsymbol{\gamma}_{k}}\right)
\end{array}
$$

where $\boldsymbol{\delta}_{k}=\Delta \varepsilon_{k}, \boldsymbol{\gamma}_{k}=\boldsymbol{g}_{k+1}-\boldsymbol{g}_{k}$ and where $\tilde{\boldsymbol{H}}_{k}$ is a symmetric matrix for which $\boldsymbol{g}_{k}^{T} \tilde{\boldsymbol{H}}_{k} \boldsymbol{g}_{k}>0$, which means that the updates $\Delta \varepsilon_{k}=-\tilde{\boldsymbol{H}}_{k} \boldsymbol{g}_{k}$ are descent directions. For the first iteration we choose $\tilde{\boldsymbol{H}}$ equal to the identity matrix. It is expected that after a sufficient number of iterations the matrices $\tilde{\boldsymbol{H}}_{k}$ become close approximations to $\boldsymbol{H}_{k}^{-1}$ [23]. Since (43) directly approximates the inverse Hessian matrix, only one matrix-vector multiplication is needed to compute the quasi-Newton correction $\Delta \varepsilon_{k}=$ $-\tilde{\boldsymbol{H}}_{k} \boldsymbol{g}_{k}$. This could be an advantage over the Gauss-Newton method, where the solution of a linear system of order $N^{\epsilon}$ [see (38) and (41)], even if this is done iteratively, can increase the computation time substantially. Unfortunately, we observed a much slower rate of convergence with this BFGS quasi-Newton implementation than with the modified Gauss-Newton implementation. This will be illustrated in the next section.

\section{ReCONSTRUCtions From SimUlated AND EXPERIMENTAL DATA}

In this section the performance of the full-wave 3-D modified Gauss-Newton reconstruction algorithm with line search is investigated for three test cases: (A) a heterogeneous lossy dielectric cube and simulated data, (B) a homogeneous dielectric sphere and exactly simulated data and (C) a homogeneous dielectric cube and experimental data. We study different antenna configurations, the effect of noise on the data and we make a comparison with the BFGS quasi-Newton algorithm with line search. All computations are performed on a computer with a 2 $\mathrm{GHz}$ Dual Core AMD Opteron processor and 8 Gbytes of RAM.

The signal-to-noise ratio (SNR) is defined as

$$
\mathrm{SNR}=10 \log _{10} \frac{\left\|e^{\text {meas }}\right\|^{2}}{2 N^{\mathrm{D}} \sigma^{2}} \mathrm{~dB}
$$

where $\sigma^{2}$ is the variance of the Gaussian white noise, which is calculated as

$$
\sigma^{2}=\frac{1}{2 N^{\mathrm{D}}-1}\left\|\boldsymbol{e}^{\mathrm{scat}}\left(\varepsilon^{0}\right)-e^{\mathrm{meas}}\right\|^{2} .
$$

Here, $\varepsilon^{0}$ is the discrete relative permittivity distribution which, within the applied discretization, yields the closest approximation to the true relative permittivity distribution. To quantify the quality of a reconstruction, we define the reconstruction error $\operatorname{err}_{\epsilon}$ as

$$
\operatorname{err}_{\epsilon}=\sqrt{\frac{1}{N^{\epsilon}} \sum_{\nu=1}^{N^{\epsilon}} \frac{\left|\epsilon_{\nu}-\epsilon_{\nu}^{0}\right|^{2}}{\left|\epsilon_{\nu}^{0}\right|^{2}}} .
$$

In all of the following, the "number of iterations" of a reconstruction refers to the number of updates of the Hessian matrix (or search direction). The total number of forward problems is larger due to the line search iterations.

\section{A. A Heterogeneous Lossy Dielectric Cube: Reconstructions From Simulated Data}

We consider a lossless dielectric cube in air, hence $\epsilon_{\mathrm{b}}=\epsilon_{0}$, with side $0.6 \lambda_{0}$ and permittivity $\epsilon=1.5 \epsilon_{0}$, which contains a smaller, lossy cube with side $0.3 \lambda_{0}$ and permittivity $\epsilon=(2-$ $2 j) \epsilon_{0}$. The origin of the reference system coincides with the center of the larger cube and the center of the smaller cube has coordinates $\left(-0.05 \lambda_{0},-0.05 \lambda_{0},-0.05 \lambda_{0}\right)$. For the inversion domain $\mathcal{D}$ we choose a cube with side $\lambda_{0}$, that is centered on the origin. Fig. 4 shows the relative complex permittivity along the three orthogonal cross sections of $\mathcal{D}$. We perform reconstructions for two antenna configurations: one yielding many and one yielding few data. In the first, "many data," configuration, shown in Fig. 5(a), there are 12 antenna positions regularly spaced on each of 6 meridional circles with radii $R=2 \lambda_{0}$. In each position, a transmitter is oriented along two polarizations, the $\theta$ - and $\varphi$-polarizations, and for each illumination the scattered field is measured along these two polarizations on receivers in all positions. In the second, "few data", configuration, shown in Fig. 5(b), only 3 circles and only the $\theta$-polarization are used. This results in $N_{1}^{\mathrm{D}}=\left(N^{\mathrm{P}}\right)^{2}\left(N^{\mathrm{A}}\right)^{2}=20736$ data for the first and $N_{2}^{\mathrm{D}}=1296$ data for the second configuration. In this paragraph, these data are simulated using the same forward solver as the one employed in the reconstruction algorithm, and the discretization grids for the inverse and forward problems coincide. This allows us to test the convergence behavior in ideal conditions. We choose $F=G=H=L=M=N=10$, hence the number of reconstruction variables is $N^{\epsilon}=1000$ and the number of field-unknowns in the forward problem is $N^{B}=$ 3300 . With the "few data" configuration the number of data thus is barely larger than the number of unknowns, $N_{2}^{\mathrm{D}} \approx N^{\epsilon}$. Moreover, there is some redundancy in the data due to reciprocity, so the "few data" configuration is actually underdetermined. The "many data" configuration is well over-determined, $N_{1}^{\mathrm{D}} \gg N^{\epsilon}$. For all reconstructions in this paragraph, the regularization parameter is chosen such that $\alpha / \mathcal{N}^{\mathcal{R}}=10^{-4}$ and the initial guess is air $\left(\epsilon^{\text {init }}=1\right)$.

Fig. 6 shows the reconstruction error $\operatorname{err}_{\epsilon}$ and the regularized cost function $\mathcal{F}$ as a function of the number of iterations. 


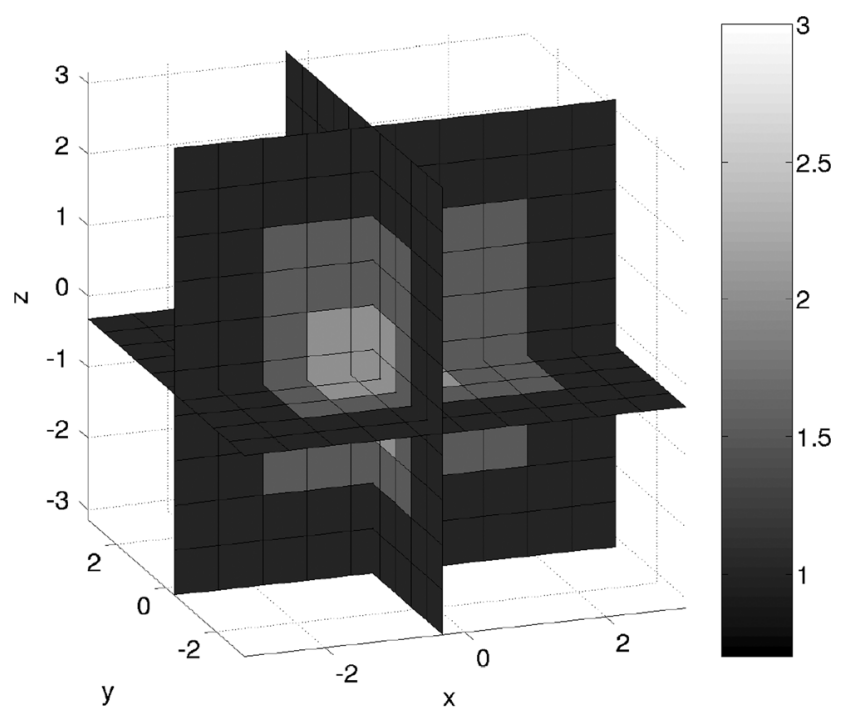

(a)

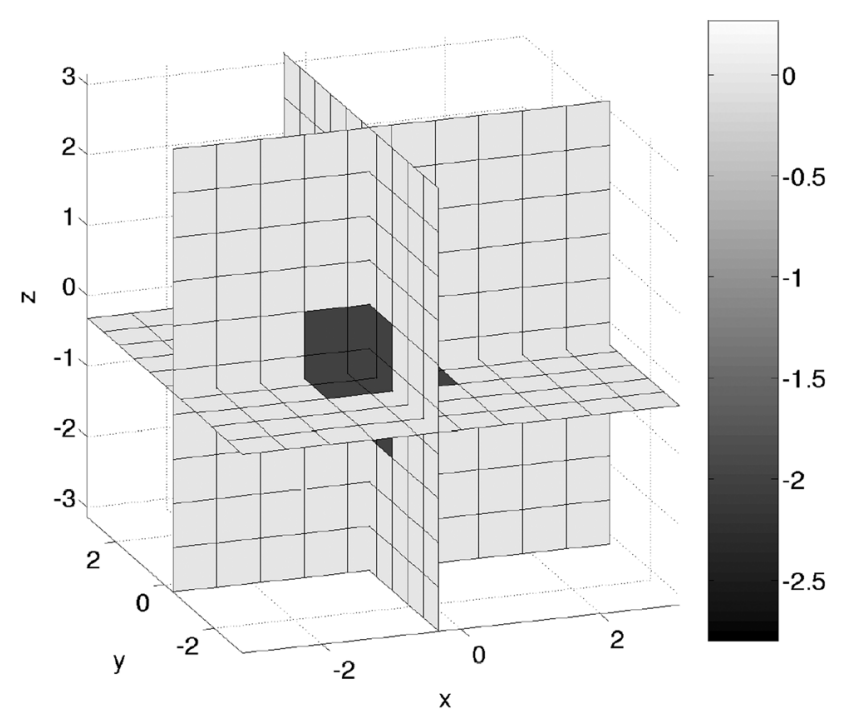

(b)

Fig. 4. The exact relative permittivity along orthogonal cross-sections of the heterogeneous lossy dielectric cube. (a) Real part and (b) imaginary part.

Let us first discuss the reconstructions from noise-free data. The threshold for the forward iterative solver BICGSTAB is set to a relative accuracy of $10^{-6}$, both for the computation of the simulated data and for the reconstructions. The reconstruction iterations are stopped when the cost function is very small, $\mathcal{F} \leq$ $10^{-8}$. It can be seen on Fig. 6(b) that the cost function rapidly decreases when applying the modified Gauss-Newton method, for both the "many data" (plain line) and "few data" (dashed line) configurations. However, Fig. 6(a) shows that the reconstruction error decreases more slowly for the "few data" configuration. After as few as 11 iterations, the "many data" configuration yields an almost exact reconstruction $\left(\operatorname{err}_{\epsilon}<0.01\right)$ of both the real and imaginary parts of the complex permittivity. This is illustrated with images along the orthogonal cross sections in Fig. 7(a) and (b). With the "few data" configuration the images and profile show small artefacts, see Fig. 7(c) and (d) and the profiles along the $x$-axis in Fig. 8, respectively.

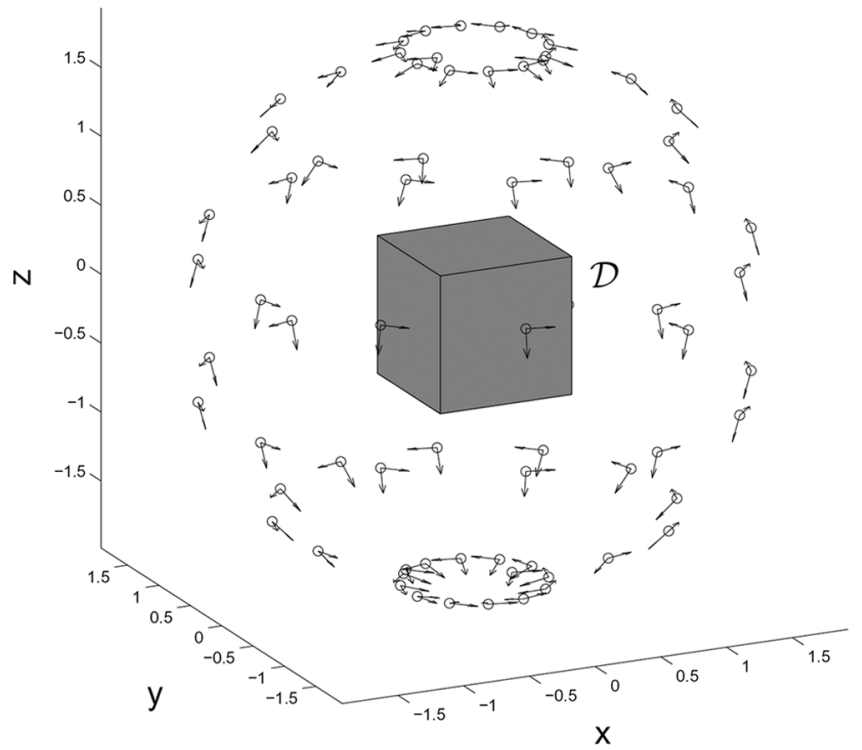

(a)

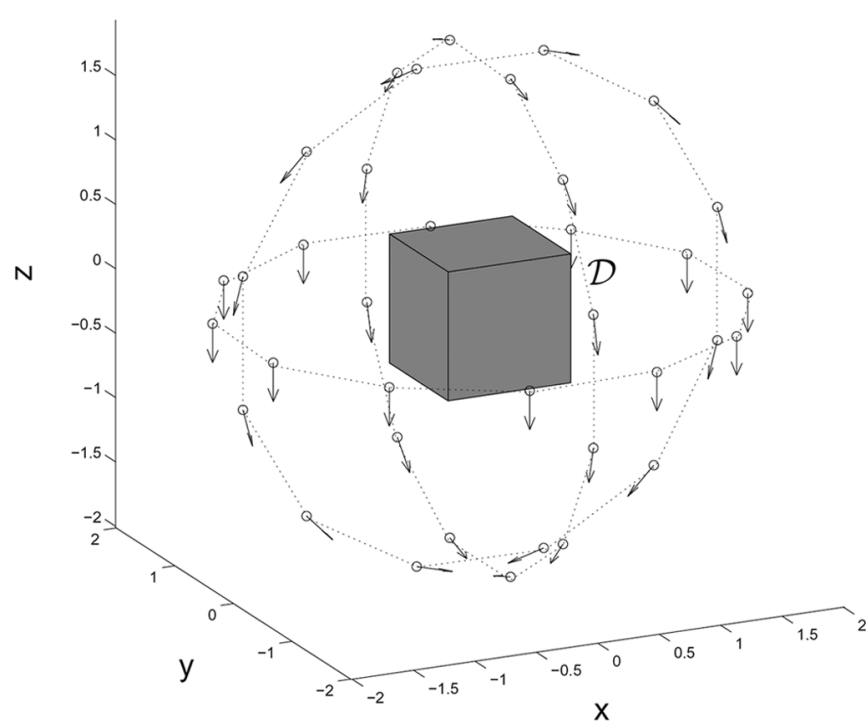

(b)

Fig. 5. The antenna configurations for the heterogeneous lossy dielectric cube. (a) Configuration 1 and (b) configuration 2.

Let us now consider data with $30 \mathrm{~dB}$ additive Gaussian noise, which corresponds to a realistic SNR, as will be seen in the following paragraphs. The threshold for the forward iterative solver BICGSTAB is now increased to $10^{-3}$, since it would be a waste of effort to solve the forward problem to an accuracy beyond the SNR. With the modified Gauss-Newton method, the reconstruction error $\operatorname{err}_{\epsilon}$ decreases and then starts to increase again at a certain point, as shown by the plain and dashed curves with crosses in Fig. 6. This happens when the data error (or least-squares cost function) reaches the noise level, i.e., $\mathcal{F}^{\mathcal{S}} \approx$ $10^{-3}$. The regularized cost function $\mathcal{F}$ hardly decreases from this point on and the tiny reductions are accompanied by an increase in the regularizing function $\mathcal{F}^{\mathcal{R}}$, which explains the rise in $\operatorname{err}_{\epsilon}$. This behavior is often called "semi-convergence." In the presence of noise on the data, we thus conclude that the stopping criteria should be based on the least squares cost function and 


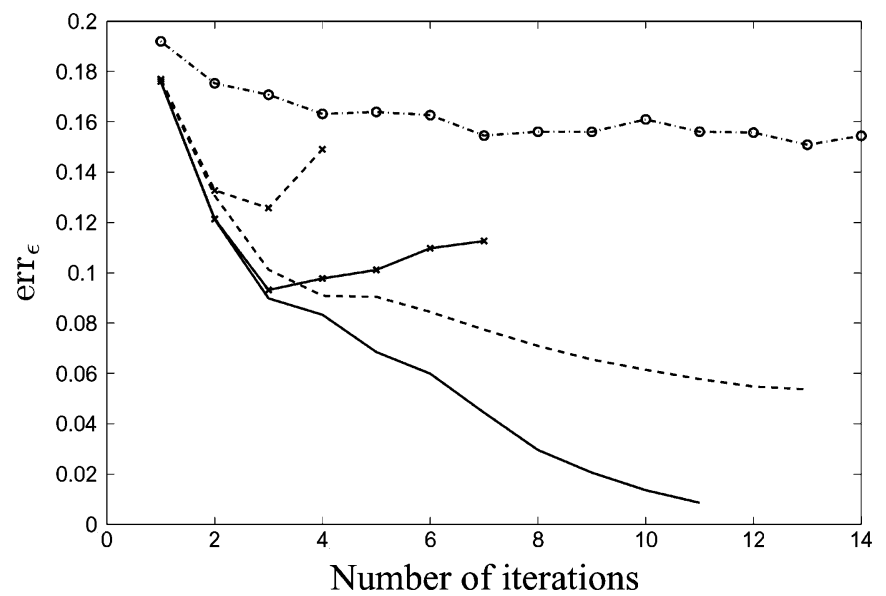

(a)

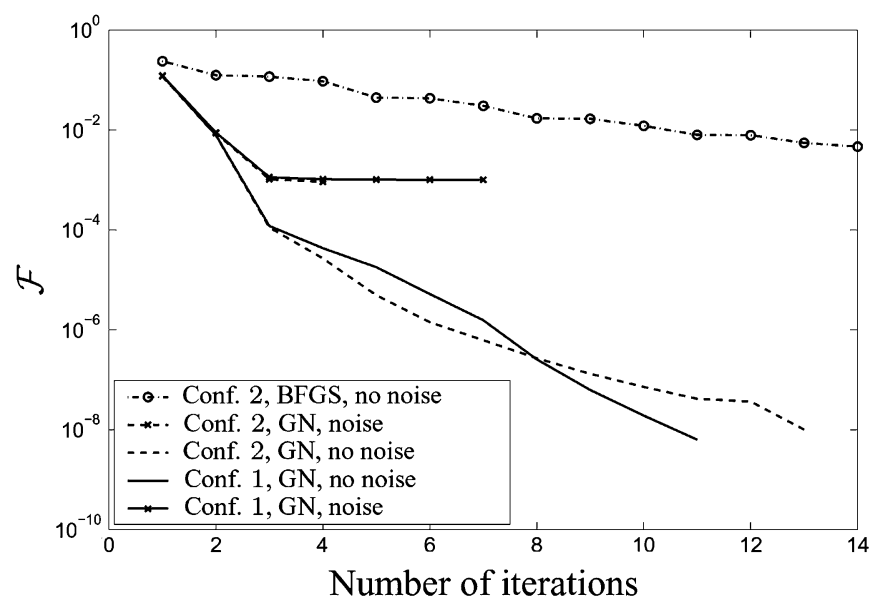

(b)

Fig. 6. The reconstruction error (a) and the regularized cost function (b) versus the number of iterations for the reconstructions of the heterogeneous lossy dielectric cube, for the "many data" (Conf. 1) and "few data" (Conf. 2) antenna configurations, with the modified Gauss-Newton (GN) and the BFGS quasiNewton (BFGS) methods using exact or noisy $(\mathrm{SNR}=30 \mathrm{~dB})$ data.

the SNR. In the examples presented here, the reconstructions thus are achieved after 3 iterations only. From the images and profile in Figs. 7(e)-(h) and 8, respectively, it appears that the noise does degrade the reconstructions, but the boundaries of the outer and inner cubes are still well reconstructed and valuable quantitative information on both the real and imaginary parts of the complex permittivity can still be retrieved. Note again that in Fig. 6 the cost-functions of both antenna configurations coincide, and that the reconstruction error behaves better for the "many data" than for the "few data" configuration.

Fig. 6 also shows a result obtained with the BFGS quasiNewton algorithm, for exact data and the "few data" configuration. Each iteration here corresponds to a Hessian update. The algorithm converges very slowly in comparison with the modified Gauss-Newton method: after 100 iterations the reconstruction error is still $10 \%$ and the cost function is reduced to $10^{-4}$ only. Such behavior was noticed in all our inversions with the BFGS-method, as well as in earlier 2-D work [11]. The advantage of not having to solve a linear system to obtain an update direction thus is completely annihilated by the large number of iterations required to converge to the desired accuracy.

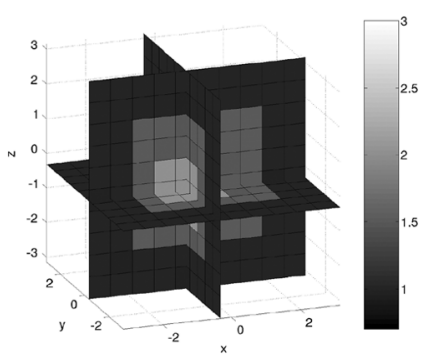

(a)

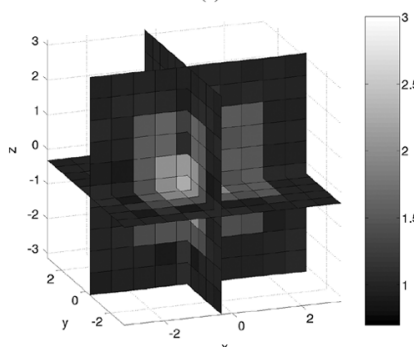

(c)

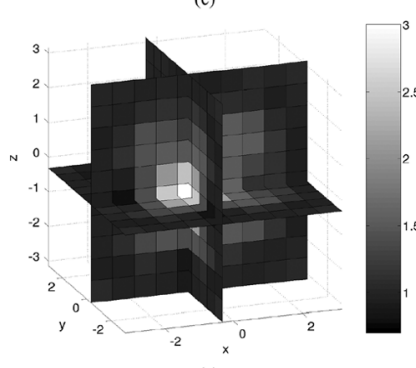

(e)

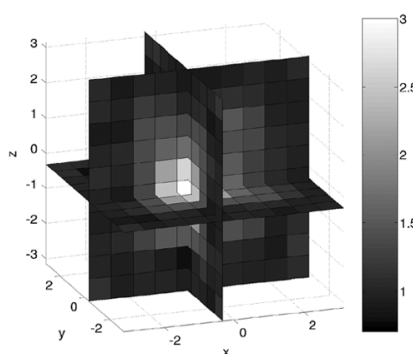

(h)

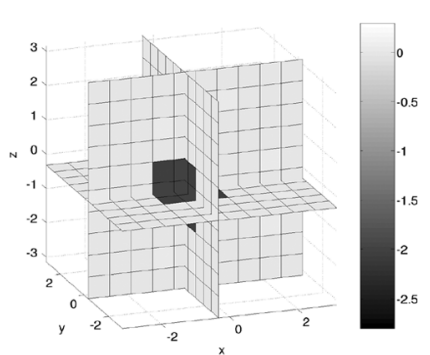

(b)

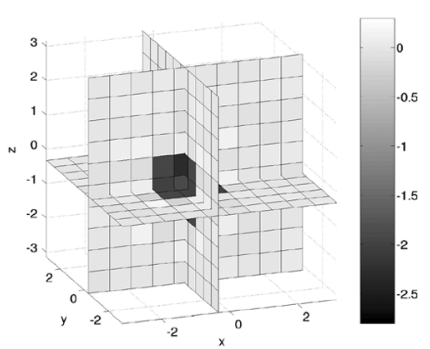

(d)

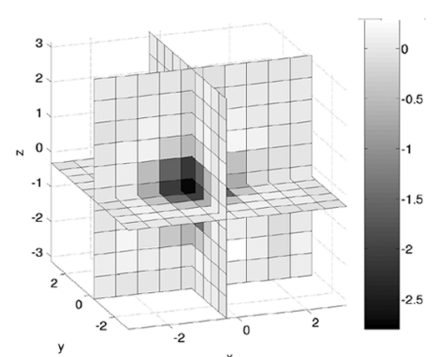

(i)

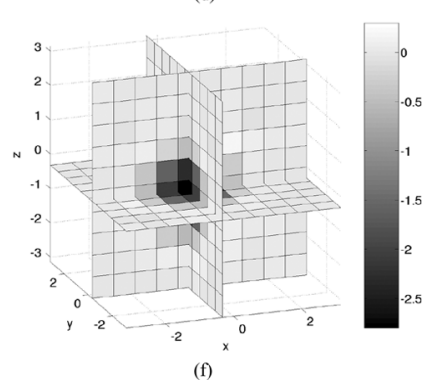

Fig. 7. Reconstructed relative permittivity corresponding to the curves in Fig. 6. (a) Conf. 1, GN, no noise, real part, (b) Conf. 1, GN, no noise, imaginary part, (c) Conf. 2, GN, no noise, real part, (d) Conf. 2, GN, no noise, imaginary part, (e) Conf. 1, GN, noise, real part, (f) Conf. 1, GN, noise, imaginary part, (h) Conf. 2, GN, noise, real part, (i) Conf. 2, GN, noise, imaginary part.

\section{B. A Homogeneous Dielectric Sphere: Reconstructions From Simulated Data}

We now consider a lossless homogeneous dielectric sphere in air with a radius $0.25 \lambda_{0}$ and permittivity $\epsilon=2 \epsilon_{0}$. The origins of the reference system and of the sphere coincide. For the inversion domain $\mathcal{D}$ we again choose a cube with side $\lambda_{0}$, that is centered on the origin. An antenna configuration identical to the "many data" configuration 1 from the previous paragraph is used, except that the radius of the meridional circles now is divided by a factor $2, R=\lambda_{0}$. The total number of scattered field data thus is again $N_{1}^{\mathrm{D}}=20736$. These data are now simulated using the full-vectorial analytic MIE solution, thus avoiding "inverse crime." We choose again the same discretization grids for the forward and inverse problems, $F=$ 


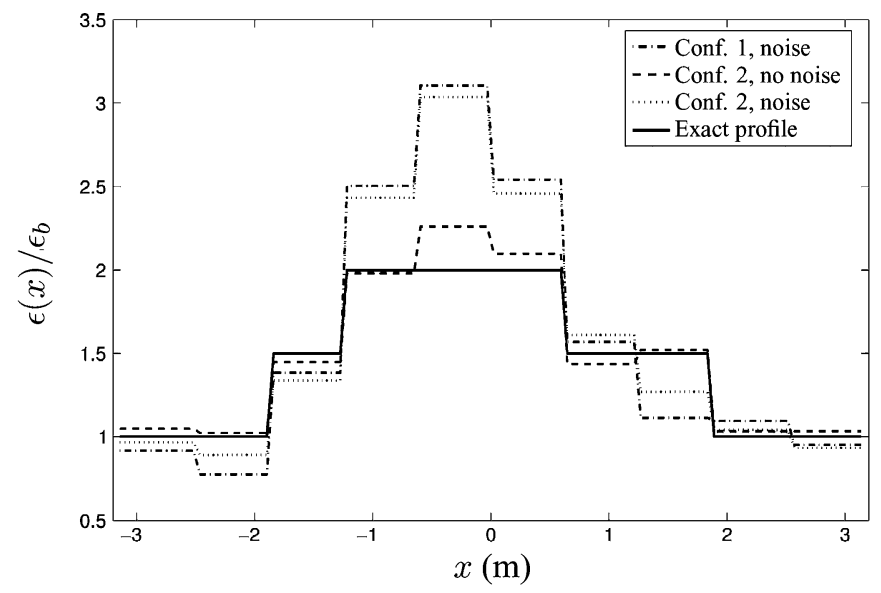

(a)

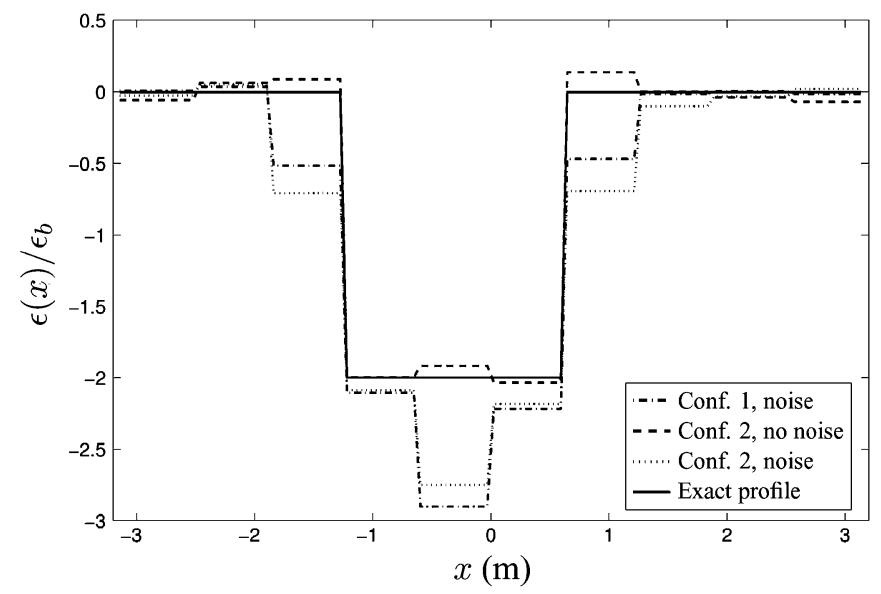

(b)

Fig. 8. Profiles of the relative permittivity along the $x$-axis for the reconstructions of Fig. 7. (a) Real part and (b) imaginary part.

$G=H=L=M=N=20$, hence the number of reconstruction variables is $N^{\epsilon}=8000$ and the number of fieldunknowns in the forward problem is $N^{B}=25200$. This discretization is actually too fine in terms of the wavelength, as $\Delta_{F}=(\lambda / 10)$ usually is recommended for a forward solution, but we did so to reduce the discretization noise introduced by the spherical boundary. This discretization noise was estimated by comparing the analytic and discretized scattered field solutions, yielding a SNR of $27 \mathrm{~dB}$. The threshold for the forward iterative solver BICGSTAB thus is set to $10^{-3}$ and the stopping criterion for the modified Gauss-Newton optimization is chosen as $\mathcal{F} \mathcal{L S} \leq 2 \cdot 10^{-3}$. The regularization parameter again satisfies $\alpha / \mathcal{N}^{\mathcal{R}}=10^{-4}$ and the initial guess is air.

Fig. 9 shows the reconstructed images along the orthogonal cross sections, obtained after as few as three iterations, comprising a total of six multiview forward problem solutions, including the line search iterations. The shape, dimensions and real part of the permittivity are well reconstructed and the imaginary part of the permittivity is small, as expected. This also appears from the profiles along the $x$-axis in Fig. 10. The total execution time for this rather large inverse problem was $1 \mathrm{~h}$ and 13 min, when using BICGSTAB iterations to solve (41) for the update directions up to a precision of $10^{-6}$.

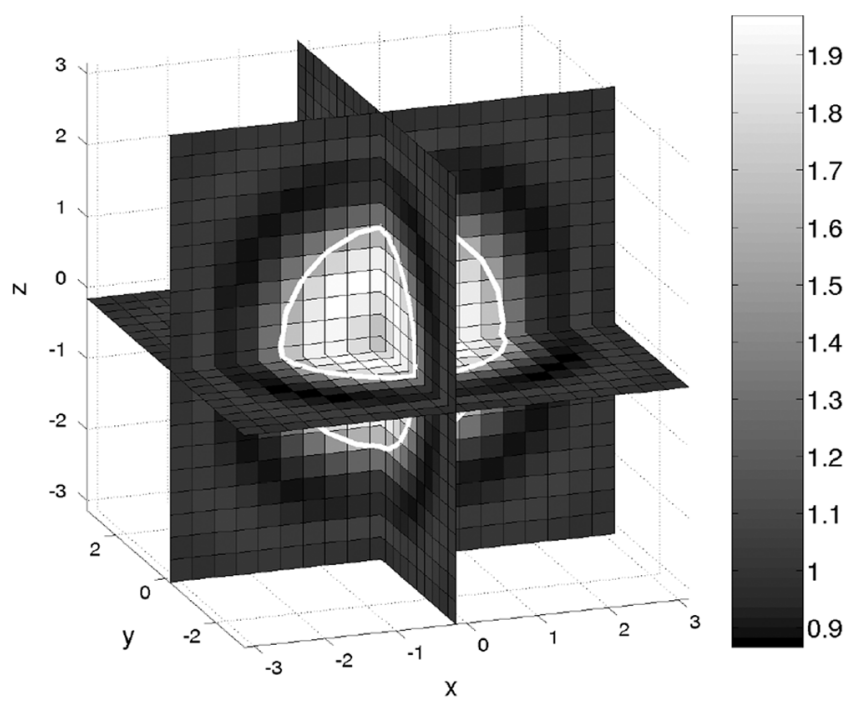

(a)

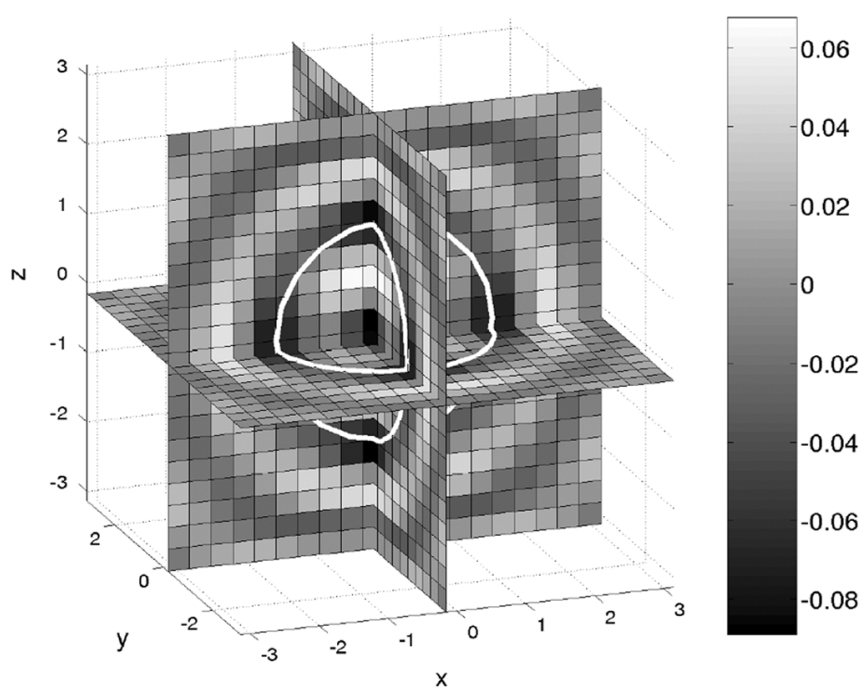

(b)

Fig. 9. Reconstructed relative permittivity after 3 iterations of a homogeneous dielectric sphere $\left(\epsilon=2 \epsilon_{0}\right)$ with radius $0.25 \lambda_{b}$ from analytic data. The white contour shows the boundaries of the actual sphere. (a) Real part and (b) imaginary part.

\section{A Homogeneous Cube: Reconstructions From Experimental Data}

We now present reconstruction results from experimental data for a homogeneous polyethylene cube with permittivity $\epsilon=2.4 \epsilon_{0}$ and side $8 \mathrm{~cm}$, surrounded with air. The scattered fields were measured in the bi-static polarimetric free-space measurement facility of Institut Fresnel, Marseille, France, as part of a first measurement campaign conducted on 3-D objects, after the successful completion of an experimental data-base for quasi-2-D objects [35]. We refer the reader to [21] for a detailed description of the Fresnel measurement set-up. In this paper, the available data are limited to measurements of the $z$-component $\left(N^{\mathrm{P}}=1\right)$ of the scattered field along an arc of a circle in the $x y$-plane with radius $R=167 \mathrm{~cm}$ and centered on the center of the cube. The $z$-polarized transmitting antenna 


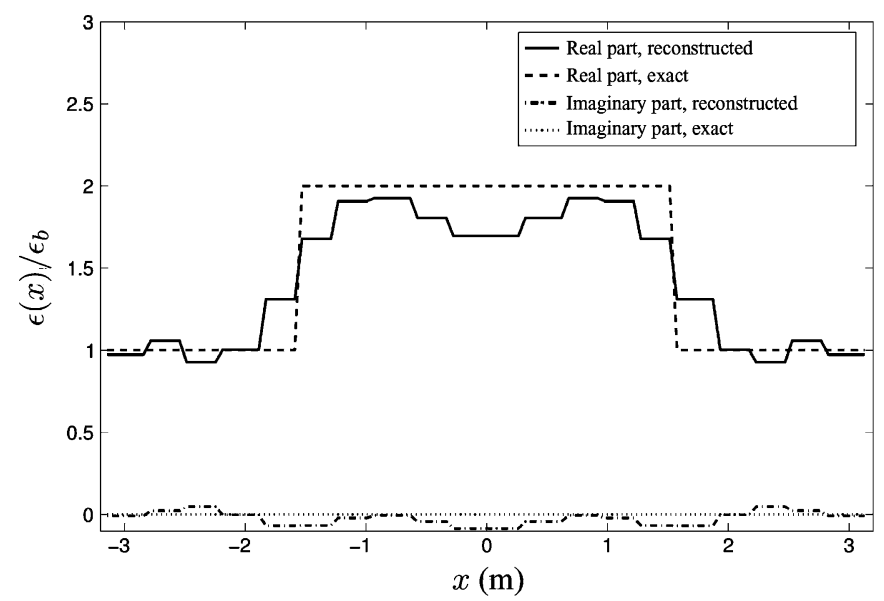

Fig. 10. Profiles of the relative permittivity along the $x$-axis for the reconstruction of Fig. 9.

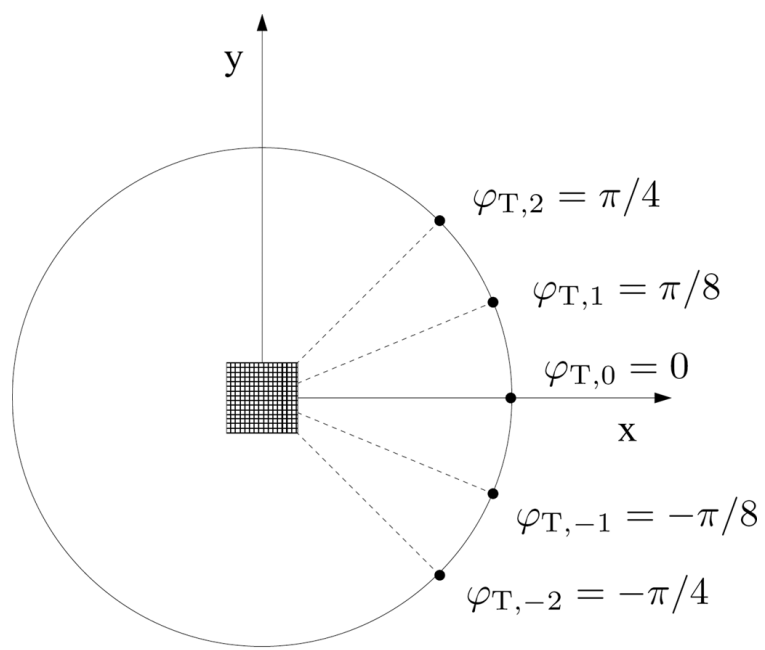

(a)

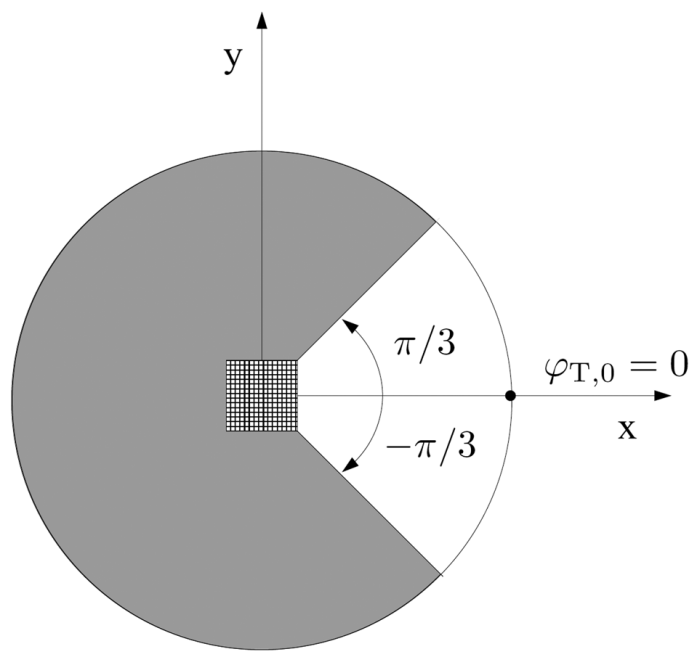

(b)

Fig. 11. Source positions (a) and range of receiver positions in gray for the source at $\varphi=0$ (b) used to collect the experimental data.

also moves on this circle. Fig. 11(a) shows the five transmitter positions, at angles $\varphi_{\mathrm{T}, k}=k \pi / 8$ for $k \in\{-2,-1,0,1,2\}$ and Fig. 11(b) shows the receiving arc for a transmitter at $\varphi_{\mathrm{T}, 0}$. For a transmitter at $\varphi_{\mathrm{T}, k}$, the receiver positions range

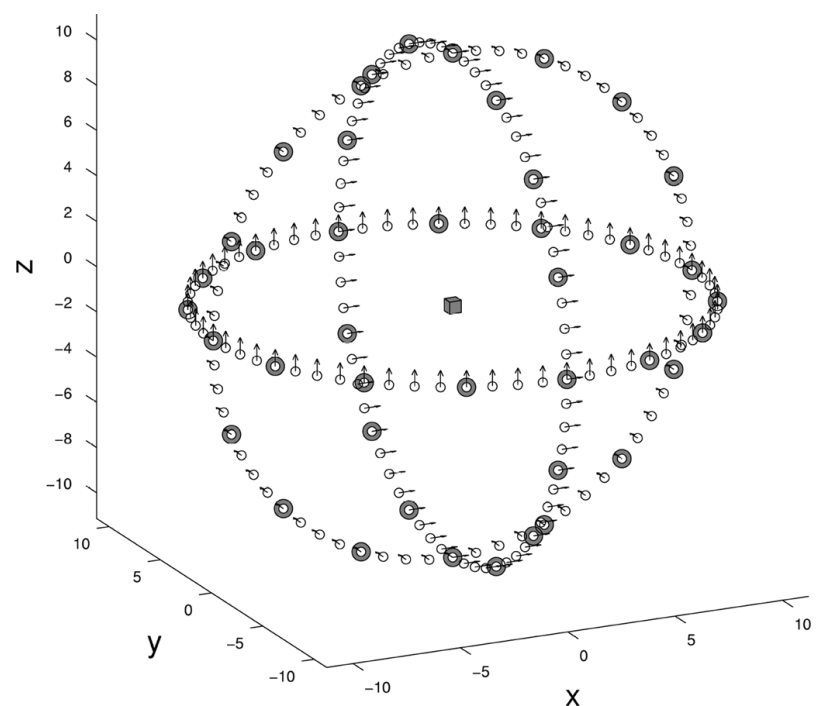

Fig. 12. Antenna configuration used for the inversion from experimental data. The encircled antennas act as both emitter and receiver.

from $\varphi_{\mathrm{T}, k}+\pi / 3$ to $\varphi_{\mathrm{T}, k}+4 \pi / 3$ in steps of 1 degree, or $\Delta \varphi_{\mathrm{R}}=\pi / 180$.

In earlier work [36] we found a good agreement between these measured data and simulations obtained with a 3-D-BICGSTAB-FFT solver for different frequencies. The transmitter was modeled as an elementary dipole and a calibration factor for each frequency was derived from the comparison of the simulated and measured incident fields in one point, i.e., in the receiver position $\varphi_{\mathrm{R}}=\pi$ opposite to the transmitter position $\varphi_{\mathrm{T}}=0$. Also, a SNR $\approx 30 \mathrm{~dB}$ at $2 \mathrm{GHz}$ and SNR $\approx 23$ $\mathrm{dB}$ at $4 \mathrm{GHz}$ was determined by comparing the calibrated measured and simulated scattered fields.

The limited data on a circle in one plane as described above are not sufficient for a successful 3-D inversion. However, since we know that the scatterer is a cube, we have exploited symmetries to extend the measurements by rotation with respect to the origin to two additional circles in the $x z$ - and $y z$-planes, and for each of the circles to extend the illuminations to 16 transmitter positions with $\Delta \varphi_{\mathrm{T}}=\pi / 8$. Of course the polarization of the antennas is rotated together with the circles. Also, for every transmitter position, we use only 43 receiver positions, with $\Delta \varphi_{\mathrm{R}}=\pi / 32$. The resulting antenna configuration is shown in Fig. 12 , where $N^{\mathrm{A}}=192, N^{\mathrm{P}}=1, N^{\mathrm{T}}=48$, and $N_{t}^{\mathrm{R}}=43$, $\forall t$.

The inversion domain $\mathcal{D}$ is a cube with side $15 \mathrm{~cm}$ and it is discretized into cells with side $\Delta_{\epsilon}=1 \mathrm{~cm}$, hence $F=$ $G=H=15$. The number of reconstruction variables thus is $N^{\epsilon}=3375$. Since the number of data $N^{\mathrm{D}}=\left(N^{\mathrm{P}}\right)^{2} N^{\mathrm{T}} N^{\mathrm{R}}$ is only 2064 , the problem is under-determined. Therefore data at two different frequencies, at 2 and $4 \mathrm{GHz}$, are used. At 2 $\mathrm{GHz}$ the grid for the forward problem coincides with the permittivity grid, $L=M=N=15$, resulting in 10800 field unknowns, but at $4 \mathrm{GHz}$ the grid for the forward problem is twice as fine, $L=M=N=30$ with $\Delta_{F}=0.5 \mathrm{~cm}$. This gives $N^{B}=83700$ field unknowns. The treshold of the BICGSTAB iterative solver is set to $10^{-3}$, in agreement with the SNR of the data. The initial estimate is again air. 

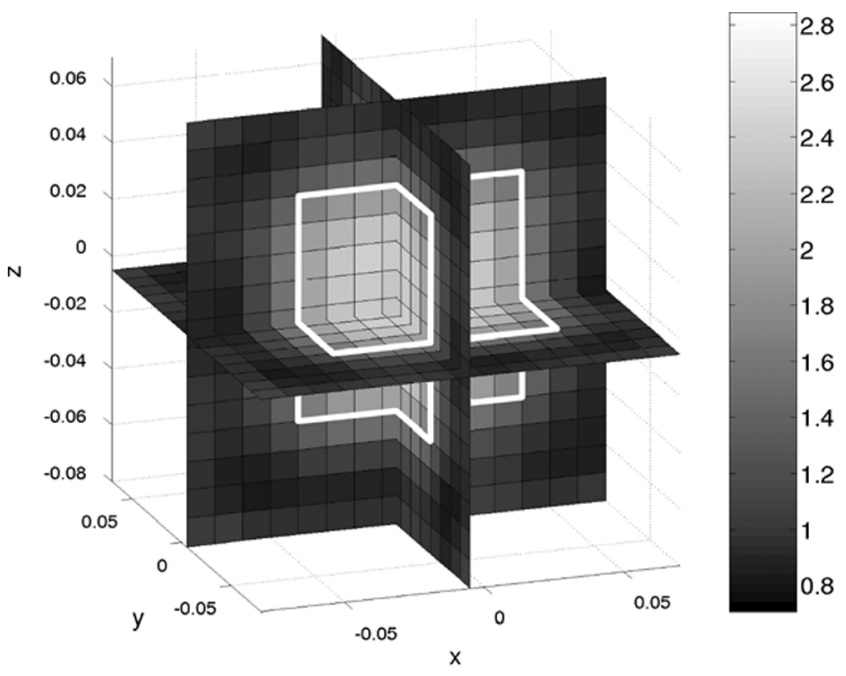

(a)
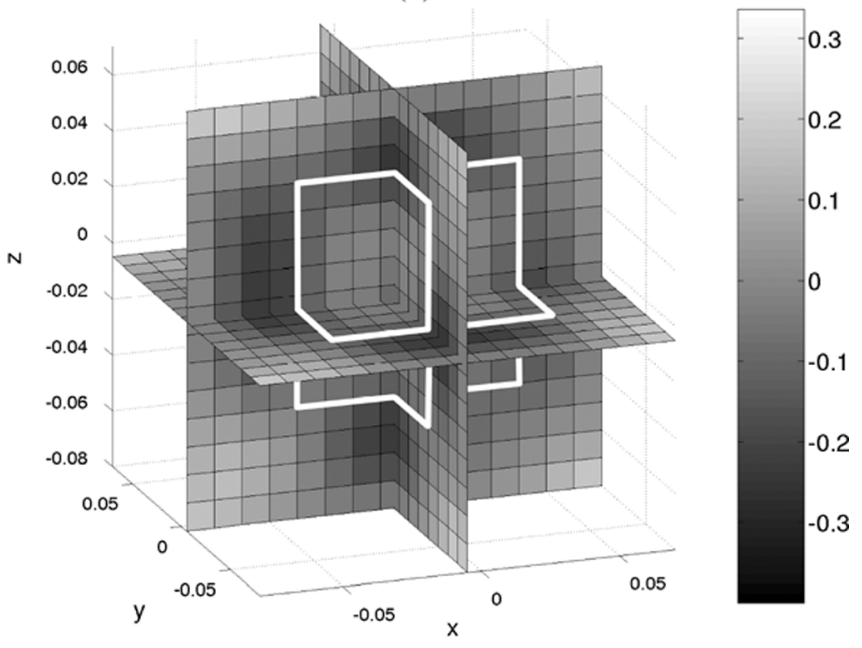

(b)

Fig. 13. Reconstructed relative permittivity of a polyethylene cube $\left(\epsilon=2.4 \epsilon_{0}\right)$ with side $8 \mathrm{~cm}$ from experimental data at $2 \mathrm{GHz}$, after 3 iterations with $\alpha / \mathcal{N}^{\mathcal{R}}=10^{-5}$. The white contour shows the boundaries of the actual polyethylene cube. (a) Real part and (b) imaginary part.

At $2 \mathrm{GHz}$ the algorithm, now with $\alpha / \mathcal{N}^{\mathcal{R}}=10^{-5}$, needed 3 iterations to reach the stopping criterion $\mathcal{F}^{\mathcal{L S}} \leq 10^{-3}$. At this point the reconstruction error was $\operatorname{err}_{\epsilon}=0.19$ and further iterations yielded no improvements. Images along the orthogonal cross sections of the reconstructions at $2 \mathrm{GHz}$ are given in Fig. 13. The total execution time was about 10 minutes and a total of six multiview forward problems was solved. Next, this result was used as an initial estimate for a subsequent reconstruction at $4 \mathrm{GHz}$ (Fig. 14), which took only 1 iteration to reach a reconstruction error of $\operatorname{err}_{\epsilon}=0.17$ and the stopping criterion $\mathcal{F}^{\mathcal{L S}} \leq 5 \cdot 10^{-3}$, which again proved to be sufficient since further iterations did not reduce $\mathrm{err}_{\epsilon}$. This time, because of the greater number of field unknowns, the execution time was much longer: about $1 \mathrm{~h}$ and $50 \mathrm{~min}$ for three multiview forward problems. Fig. 15 displays the evolution of the reconstruction error along the complete reconstruction path and Fig. 16 shows the relative permittivity profiles along the $x$-axis. Although only limited experimental data is used in this example, the modified Gauss-Newton method yields a promising result. When more

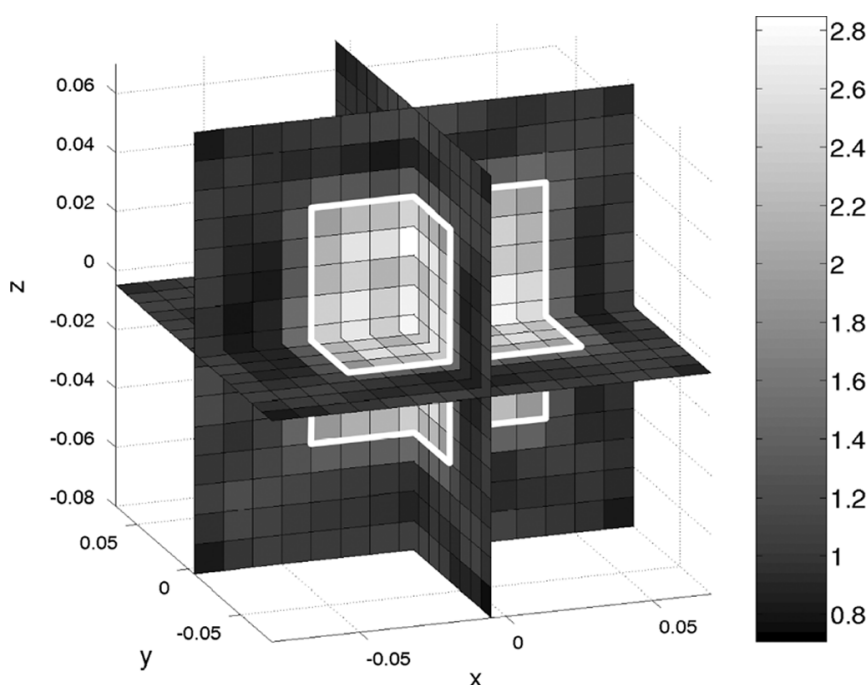

(a)

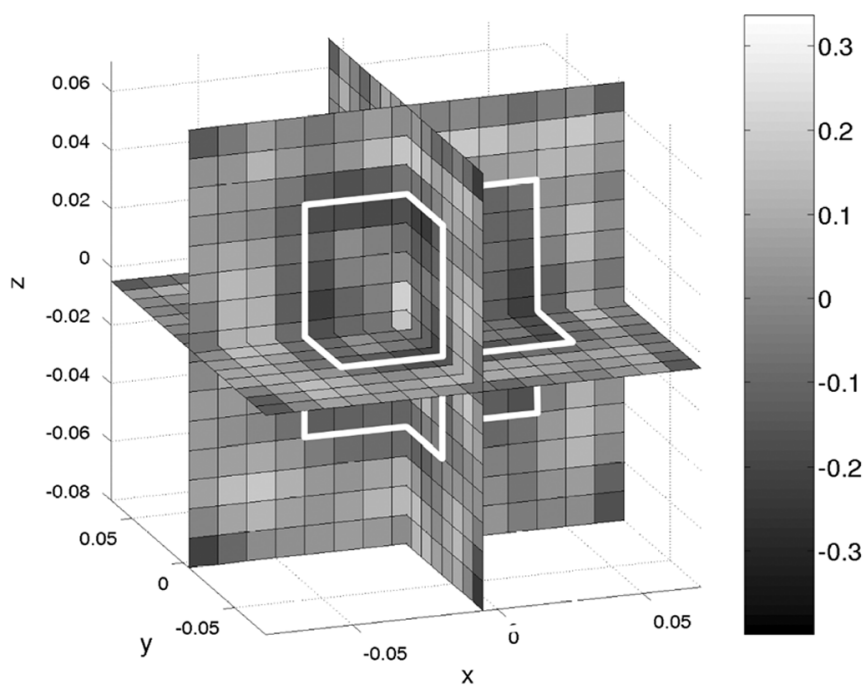

(b)

Fig. 14. Reconstructed relative permittivity, starting from the result of Fig. 13, after 1 iteration at $4 \mathrm{GHz}$ and with $\alpha / \mathcal{N}^{\mathcal{R}}=10^{-5}$. The white contour shows the boundaries of the actual polyethylene cube. (a) Real part and (b) imaginary part.

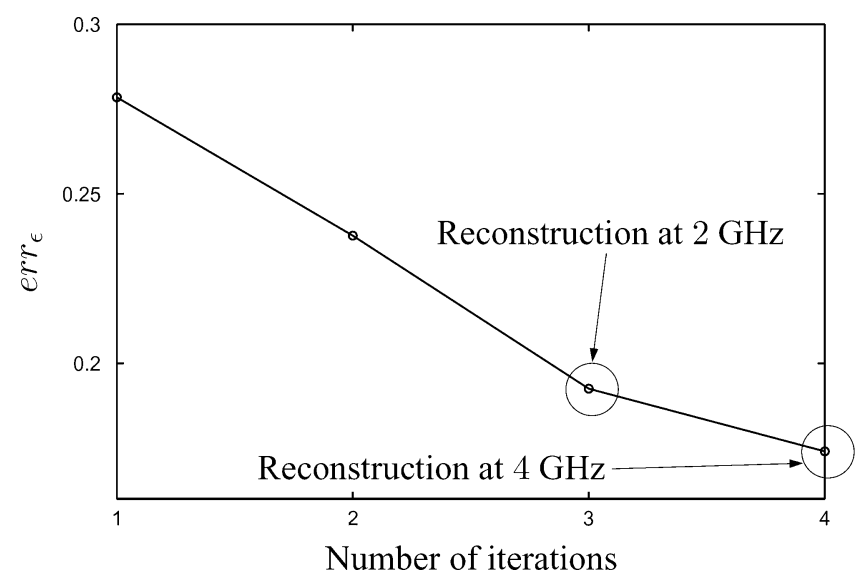

Fig. 15. Evolution of the reconstruction error for the inversion, corresponding to Figs. 13 and 14.

complete scattering data become available, the quality of the reconstructions could even further improve. 


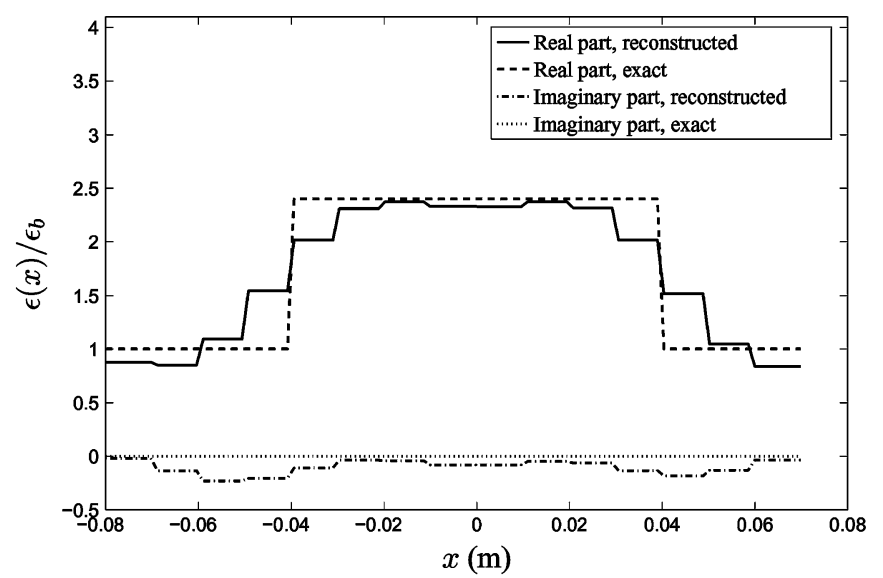

(a)

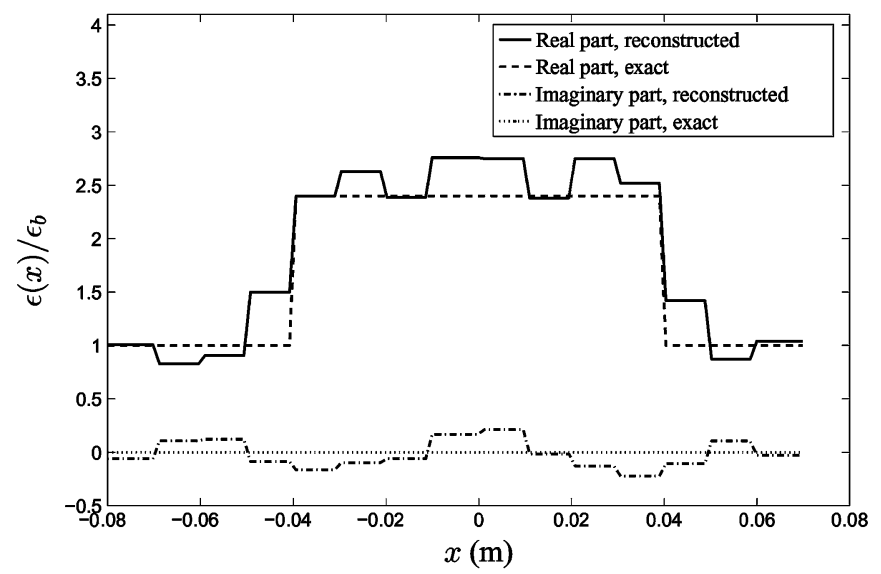

(b)

Fig. 16. Profiles of the relative permittivity along the $x$-axis for the reconstructions of a homogeneous dielectric cube from experimental data, at $2 \mathrm{GHz}$ (a) and $4 \mathrm{GHz}(\mathrm{b})$.

\section{CONCLUSION}

The problem of 3-D complex permittivity reconstruction from vectorial scattered field measurements was addressed as a non-linear optimization problem. A multiplicative-additive regularization was applied to the least-squares cost function, in which only the permittivity was considered as the unknown. As a consequence, a multiview forward scattering problem had to be solved in each iteration of the optimization algorithm. This was accomplished in a computationally efficient way by the combination of a stable iterative solver (BICGSTAB), a fast multiplication scheme (the FFT-method) and an extrapolation procedure to find good initial estimates (the marching-on-in-source-position technique). The regularized cost function was minimized using a modified Gauss-Newton approach with a line search. This method appeared to converge much faster than the BFGS quasi-Newton method. It was shown that the multiplicative-additive regularization improved the conditioning of the linear subproblems in the non-linear optimization, that it mitigated the effect of noise on the reconstruction and that it dealt in a more efficient way with the non-linearity of the inverse scattering problem. Numerical examples on both simulated and experimental data illustrated the effectiveness of the proposed inversion method. Future work will focus on reducing the computational cost of the algorithm, which remains the major disadvantage of pixel-based quantitative inversion methods.

\section{REFERENCES}

[1] W. C. Chew and Y. M. Wang, "Reconstruction of two-dimensional permittivity distribution using the distorted born iterative method," IEEE Trans. Med. Imaging, vol. 9, no. 2, 1990.

[2] N. Joachimowicz, C. Pichot, and J. Hugonin, "Inverse scattering: An iterative numerical method for electromagnetic imaging," IEEE Trans. Antennas Propag., vol. 39, no. 12, pp. 1742-1752, 1991.

[3] L. Garnero, A. Franchois, J. P. Hugonin, C. Pichot, and N. Joachimowicz, "Microwave imaging-Complex permittivity reconstruction by simulated annealing," IEEE Trans. Microw. Theory Tech., vol. 39, pp. 1801-1807, 1991.

[4] R. E. Kleinman and P. M. van den Berg, "A modified gradient method for two-dimensional problems in tomography," J. Comput. Appl. Math., vol. 42, no. 1, pp. 17-35, 1992.

[5] A. Franchois and C. Pichot, "Microwave imaging-Complex permittivity reconstruction with a Levenberg-Marquardt method," IEEE Trans. Antennas Propag., vol. 45, no. 2, pp. 203-215, 1997.

[6] P. M. van den Berg and R. E. Kleinman, "A contrast source inversion method," Inverse Problems, vol. 13, no. 6, pp. 1607-1620, 1997.

[7] S. Caorsi, A. Massa, and M. A. Pastorino, "A computational technique based on a real-coded genetic algorithm for microwave imaging purposes," IEEE Trans. Geosci. Remote Sensing, vol. 38, no. 4, pp. 1697-1708, 2000.

[8] A. G. Tijhuis, K. Belkebir, and A. C. S. Litman, "Theoretical and computational aspects of 2-D inverse profiling," IEEE Trans. Geosci. Remote Sensing, vol. 39, no. 6, pp. 1316-1330, 2001.

[9] A. Abubakar, T. M. Habashy, P. M. van den Berg, and D. Gisolf, "The diagonalized contrast source approach: An inversion method beyond the born approximation," Inverse Problems, vol. 21, no. 2, pp. 685-702, 2005.

[10] T. J. Cui, W. C. Chew, A. A. Aydiner, and S. Chen, "Inverse scattering of two-dimensional dielectric objects buried in a lossy earth using the distorted born iterative method," IEEE Trans. Geosci. Remote Sensing, vol. 39, no. 2, pp. 339-345, 2001.

[11] A. Franchois and A. G. Tijhuis, "A Quasi-Newton reconstruction algorithm for a complex microwave imaging scanner environment," Radio Sci., vol. 38, no. 2, 2003.

[12] A. Franchois, A. Joisel, C. Pichot, and J. C. Bolomey, "Quantitative microwave imaging with a $2.45 \mathrm{GHz}$ planar microwave camera," IEEE Trans. Med. Imaging, vol. 17, no. 4, pp. 550-561, 1998.

[13] P. M. Meaney, K. D. Paulsen, and J. T. Chang, "Nonactive antenna compensation for fixed-array microwave imaging: Part II-Imaging results," IEEE Trans. Med. Imaging, vol. 18, no. 6, pp. 508-518, 1999.

[14] O. Franza, N. Joachimowicz, and J. C. Bolomey, "SICS: A sensor interaction compensation scheme for microwave imaging," IEEE Trans. Antennas Propag., vol. 50, no. 2, pp. 211-216, 2002.

[15] A. E. Bulyshev, A. E. Souvorov, S. Y. Semenov, R. H. Svenson, A. G. Nazarov, Y. E. Sizov, and G. P. Tatsis, "Three-dimensional microwave tomography. Theory and computer experiments in scalar approximation," Inverse Problems, vol. 16, no. 3, pp. 863-875, 2000.

[16] A. Abubakar, P. M. van den Berg, and J. J. Mallorqui, "Imaging of biomedical data using a multiplicative regularized contrast source inversion method," IEEE Trans. Microw. Theory Tech., vol. 50, no. 7, pp. 1761-1770, 2002.

[17] Z. Q. Zhang and Q. H. Liu, "Three-dimensional nonlinear image reconstruction for microwave biomedical imaging," IEEE Trans. Biomed. Eng., vol. 51, no. 3, pp. 544-548, 2004.

[18] G. Franceschini, D. Franceschini, and A. Massa, "Full-vectorial three-dimensional microwave imaging through the iterative multiscaling strategy-A preliminary assessment," IEEE Trans. Geosci. Remote Sensing Letters, vol. 2, no. 4, pp. 428-432, 2005.

[19] K. Belkebir, P. C. Chaumet, and A. Sentenac, "Influence of multiple scattering on three-dimensional imaging with optical diffraction tomography," J. Opt. Soc. Am. A., vol. 23, no. 3, pp. 586-595, 2006.

[20] T. Takenaka, H. Zhou, and T. Tanaka, "Inverse scattering for a threedimensional object in the time domain," J. Opt. Soc. Am. A, vol. 20, no. 10, pp. 1867-1874, 2003.

[21] J. M. Geffrin, P. Sabouroux, and C. Eyraud, "Free space experimental scattering database continuation: Experimental set-up and measurement precision," Inverse Problems, vol. 21, pp. S117-S130, 2005.

[22] P. Zwamborn and P. M. van den Berg, "The three-dimensional weak form of the conjugate gradient FFT method for solving scattering problems," IEEE Trans. Microw. Theory Tech., vol. 40, no. 9, pp. 1757-1766, 1992.

[23] R. Fletcher, Practical Methods of Optimization, 2nd ed. New York: John Wiley, 1990.

[24] J. Van Bladel, Electromagnetic Fields. New York: Springer-Verlag, 1985. 
[25] J. De Zaeytijd, I. Bogaert, and A. Franchois, "An efficient hybrid MLFMA-FFT solver for the volume integral equation in case of sparse 3-D inhomogeneous dielectric scatterers," J. Comput. Phys., submitted for publication.

[26] A. Kirsch, An Introduction to the Mathematical Theory of Inverse Problems. New York: Springer-Verlag, 1996.

[27] R. D. da Cunha and T. Hopkins, "The parallel iterative methods (PIM) package for the solution of systems of linear equations on parallel computers," Appl. Num. Math., vol. 19, no. 1-2, pp. 33-50, 1995.

[28] H. Gan and W. C. Chew, "A discrete BCG-FFT algorithm for solving 3-D inhomogeneous scatterer problems," IEEE Trans. Antennas Propag., vol. 9, no. 10, pp. 1339-1357, 1995.

[29] T. K. Sarkar, E. Sarvas, and S. M. Rao, "Application of FFT and the conjugate gradient method for the solution of electromagnetic radiation from electrically large and small conducting bodies," IEEE Trans. Antennas Propag., vol. 34, no. 5, pp. 635-640, 1986.

[30] Z. Q. Peng and A. G. Tijhuis, "Transient scattering by a lossy dielectric cylinder: Marching-on-in-frequency approach," J. Electrom. Waves. Applic., vol. 7, no. 5, pp. 739-763, 1993.

[31] A. Abubakar and P. M. van den Berg, "Total variation as a multiplicative constraint for solving inverse problems," IEEE Trans. Image Process., vol. 10, no. 9, 2001

[32] V. A. Morozov, "On the solution of functional equations by the method of regularization," Soviet Math. Dokl., vol. 7, pp. 414-417.

[33] A. van den Bos, "Complex gradient and hessian," Proc. Inst. Elect. Eng. Vision, Image Signal Process., vol. 141, no. 6, pp. 380-383, 1994.

[34] A. N. Tikhonov and V. Y. Arsenine, Solutions of Ill-Posed Problems. Washington, DC: Winston, 1977.

[35] K. Belkebir and M. Saillard, "Testing inversion algorithms against experimental data: Inhomogeneous targets," Inverse Problems, vol. 21, no. 6, pp. S1-S3, 2005.

[36] P. Lewyllie, A. Franchois, C. Eyraud, and J. M. Geffrin, "Testing a 3-D BCGS-FFT solver against experimental data," in Proc. ICEAA'05, 2005, pp. 421-424.

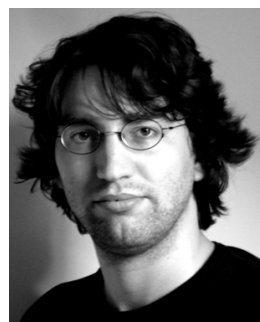

Jürgen De Zaeytijd was born in Oudenaarde, Belgium, in 1981. He received the M.S. degree in physical engineering from Ghent University, Ghent, Belgium, in 2004. His thesis dealt with the simulation of electromagnetic scattering from electrically large two dimensional scatterers by means of the fast multipole method.

In October 2004, he joined the Electromagnetics Group of the Department of Information Technology (INTEC-IMEC) at Ghent University, Belgium. His research, which is funded by an FWO doctoral grant, is focused on the exact inverse scattering problem using microwaves.

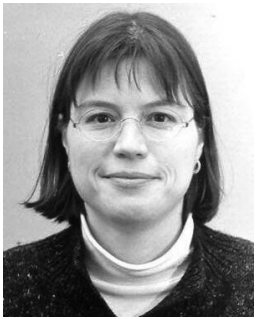

Ann Franchois (M'96-SM'99) was born in Geel, Belgium, in 1964. She received the M.S. degree in electrical engineering from the Ghent University, Belgium, in 1988, and the DEA and Ph.D. degrees in optics and photonics from the University of Paris-Sud, Orsay, France, in 1989 and 1993, respectively.

From 1994 to 1996, she was with the Research Division of Alcatel Bell Telephone, Antwerp, Belgium, working on EMC and from 1996 to 1999, she was with the Joint Research Center of the Commission of the European Communities, Ispra, Italy, working on microwave sensing applications. From 1999 to 2000, she held a Postdoctoral Teaching-Research position in the Electromagnetics Group, Faculty of Electrical Engineering at the Eindhoven University of Technology, The Netherlands. In 2000, she joined the Department of Information Technology (INTEC-IMEC) at Ghent University, Belgium, where she is an Associate Professor. Her research interests include inverse scattering and imaging.

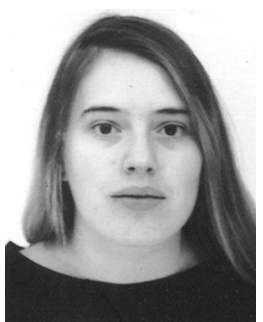

Christelle Eyraud received the Ph.D. degree from the University of Provence, France, in 2006.

She worked at the Institut Fresnel, Université Paul Cézanne Aix-Marseille, III, Ecole Généraliste d'Ingénieurs de Marseille, Université de Provence Aix-Marseille I, France, on experimental developments and measurement errors analysis for microwave scattering phenomena. After a Postdoctoral position at the laboratory PALMS, Université de Rennes 1, France, she is currently working at the Laboratoire de Planétologie, Grenoble, France in a

Postdoctoral position.

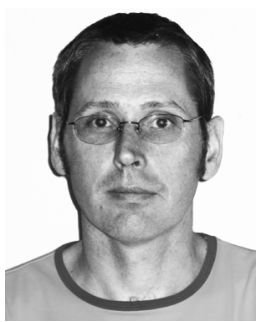

Jean-Michel Geffrin received the Ph.D. degree in physics from the University of Paris XI, France, in 1993.

He worked for ten years as a Research Engineer at the Laboratoire des Signaux et Systèmes, Gif-sur-Yvette, France, where he developed specific antennas and experimental setups for measuring targets radiation pattern. In 2002, he joined the Institut Fresnel, Université Paul Cézanne Aix-Marseille, III, Ecole Généraliste d'Ingénieurs de Marseille, Université de Provence Aix-Marseille I, France, to reinforce the hyperfrequency experimentalist team and has contributed to the constitution of the second database of scattered fields proposed by the Institut Fresnel to the inverse problem community. 